\title{
ON THE DYNAMICS OF A CLOSED THERMOSYPHON
}

\author{
By
}

\section{J.J.L. Velázquez}

IMA Preprint Series \# 1080

December 1992 


\title{
ON THE DYNAMICS OF A CLOSED THERMOSYPHON
}

\author{
by
}

\section{J.J.L. Velázquez}

\section{INTRODUCTION}

In this work we shall study the motion of an incompressible fluid at the interior of a closed loop under the effect of convective forces arising from differences of temperature. We will assume that the section of the pipe is constant and small, so that the motion of the fluid can be supposed to be one-dimensional. Then the equations describing conservation of mass and momentum are given respectively by

$$
\begin{gathered}
\frac{\partial \rho}{\partial \tau}+\frac{\partial}{\partial s}(\rho u)=0 \\
\frac{\partial u}{\partial \tau}+\frac{1}{2} \frac{\partial}{\partial s} u^{2}+\frac{1}{\rho} \frac{\partial p}{\partial s}+\frac{\partial U}{\partial s}=-\frac{\lambda|u| u}{2 D}
\end{gathered}
$$

where $s$ is the length coordinate along the loop and $\tau$ is the time coordinate: $\rho(s, \tau), p(s, \tau)$, and $u(s, \tau)$ denote respectively the density, pressure and velocity of the fluid. $U(s)$ represents the potential corresponding to gravitational forces, so that $f_{m}=-\frac{\partial U}{\partial s}, f_{m}(s)$ being the component of gravitational forces along the loop by unit of mass. $D$ stands for the diameter of the pipe, whereas $\lambda$ is Darcy-Weissbach coefficient. Using standard thermodynamical relations, we also obtain an equation for the enthropy $S(s, \tau)$, namely

$$
\theta\left(\frac{\partial S}{\partial \tau}+v \frac{\partial S}{\partial s}\right)=\frac{\lambda u^{3}}{2 D}+\frac{4 q_{s}}{\rho D}
$$

where $\theta=\theta(s, \tau)$ is the temperature of the fluid and $q_{s}$ is the heat transfer coefficient at the wall. As it is often done when dealing with convective motions in liquids, we shall make use of the Boussinesq approximation here. We therefore will assume that density is constant in the continuity equation (1.1) (which yields $u=u(\tau)$ ), and consider $\rho$ in the forms $\rho=\rho_{0}\left(1-\left(\theta-\theta_{0}\right)\right)$ in (1.2), where $\rho_{0}$ is constant, $\theta_{0}$ is some mean temperature, and $\left(\theta-\theta_{0}\right)<<1$, so that variations of temperature are assumed to be small. (Here and henceforth we shall make use of the customary asymptotic notations $O, o,<<$, etc.). Furthermore, one then supposes that variations of pressure are very small when compared with the hydrostatic pressure at equilibrium, $p_{h}$. If we then write $p=p_{h}+\tilde{p}$, where $\tilde{p}<<p_{h}$, and notice that $\frac{1}{\rho_{0}} \frac{\partial p_{h}}{\partial s}+\frac{\partial U}{\partial s}=0$, it readily follows that, retaining only lower order terms, 


$$
\frac{1}{\rho} \frac{\partial \tilde{p}}{\partial s}+\frac{\partial U}{\partial s}=\frac{1}{\rho_{0}} \frac{\partial \tilde{p}}{\partial s}+\left(\theta-\theta_{0}\right) f_{m}
$$

We substitute this equation in (1.2), and assume that the loop geometry is described by a function $\tilde{z}=\tilde{z}(s)$, so that $f_{m}=-g \frac{d \tilde{z}}{d s}$. Integrating then the resulting equation along the loop, whose total length is denoted by $L$, we arrive at

$$
L \frac{d u}{d \tau}=g \int_{0}^{L}\left(\theta-\theta_{0}\right) \tilde{z}^{\prime}(s) d s-\frac{\lambda L}{\tau D}|u| u
$$

In the enthropy equation, we suppose that $q_{s}=\frac{\lambda \rho v}{8} c_{\ell}\left(\theta_{w}-\theta\right)$ where $c_{\ell}$ is the specific heat of the liquid and $\theta_{w}$ is the temperature at the wall (Reynolds analogy). Assuming that $u^{2}<<c_{\ell}\left(\theta_{w}-\theta\right)$, we obtain, after setting $S=c_{\ell} \log \theta+s_{0}$ for some constant $s_{0}$

$$
\frac{\partial \theta}{\partial \tau}+u(\tau) \frac{\partial \theta}{\partial s}=\frac{\lambda|u|}{2 D}\left(\theta_{w}-\theta\right)
$$

We now turn our attention to the Darcy-Weissbach coefficient $\lambda$, which is a function of the Reynolds number

$$
R e=\frac{v_{c} D}{\nu},
$$

where $v_{c}$ is a characteristic velocity of the problem and $\nu$ is the viscosity coefficient. Thus $\lambda=\lambda(\tilde{R} e)$, where function $\lambda(\xi)$ is usually in the form

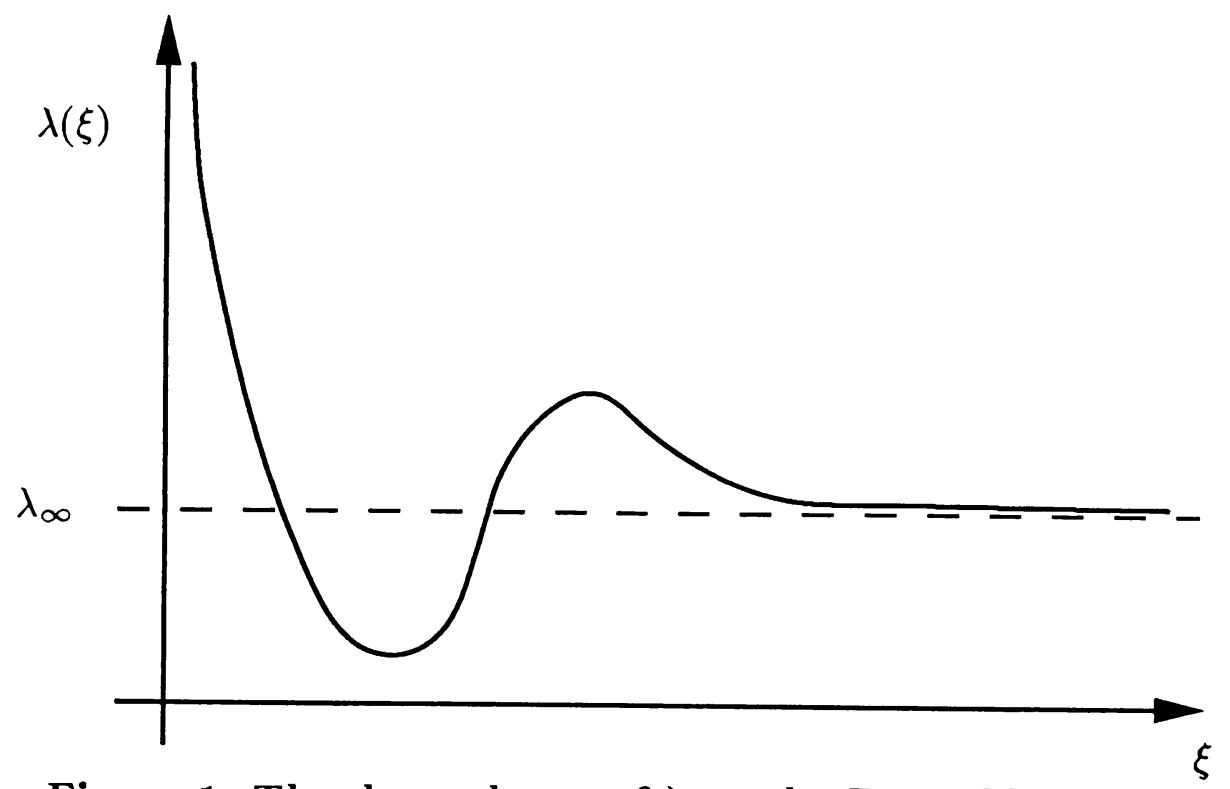

Figure 1: The dependence of $\lambda$ on the Reynolds number 
(cf. for instance $[P T]$, Chapter III, Section $B$ ). More precisely, we shall assume that

$$
\begin{gathered}
\lambda(\xi) \approx \frac{A_{1}}{\xi} \text { as } \xi \downarrow 0 \text { for some } A_{1}>0 \\
\lambda(\xi) \rightarrow \lambda_{\infty} \text { as } \xi \rightarrow \infty \text { for some } \lambda_{\infty}>0
\end{gathered}
$$

Additional requirements on $\lambda(\xi)$ will be made explicit later when required. We now define a new set of non-dimensional variables by setting

$$
t=\frac{\tau}{t_{0}}, \quad x=\frac{s}{L}, \quad v=\frac{u}{v_{c}}, \quad z=\frac{\tilde{z}}{L}, \quad T=\frac{\theta-\theta_{0}}{\theta_{c}}
$$

where $\theta_{c}$ is some characteristic temperature, $v_{c}=\left\{\frac{2 g \theta_{c} D}{g}\right\}^{1 / 2}$, and $t_{0}=\frac{L}{v_{c}}$. Putting $\epsilon=\frac{2 D}{\lambda_{\infty} L}$ and $T_{w}=\frac{\theta_{w}-\theta_{0}}{\theta_{c}}$, we obtain that (1.4) and (1.5) give

$$
\epsilon \frac{d v}{d t}=\int_{0}^{1} T(x, t) z^{\prime}(x) d x-\frac{\lambda}{\lambda_{\infty}}|v| v
$$

$$
\frac{\partial T}{\partial t}+v \frac{\partial T}{\partial x}=\frac{\lambda|v|}{\epsilon \lambda_{\infty}}\left(T_{w}-T\right)
$$

We finally define

$$
g(\xi)=\frac{\lambda(\xi)}{\lambda_{\infty}}
$$

so that $\frac{\lambda}{\lambda_{\infty}} \frac{|u| D}{\nu}=g\left(\frac{v_{c} D}{\nu}|v|\right)$. Therefore, $g$ is such that

$$
g(\xi) \approx \frac{A}{\xi} \text { as } \xi \downarrow 0 \text { for some } A>0,
$$

(compare with Figure 1). We shall denote by $R e$ the associated Reynolds number given by

$$
\lim _{\xi \rightarrow \infty} g(\xi)=1
$$

$$
R e=\frac{v_{c} D}{\nu}
$$


We shall refer henceforth to Re as the Reynolds number for short. If we now write $f(x)=z^{\prime}(x)$, we are thus led to the study of the following

Problem.- To find $v(t)$ and $T(x, t)$ such that

$$
\epsilon \frac{d v}{d t}=\oint T(x, t) f(x) d x-g(\operatorname{Re}|v|)|v| v \text { for } t>0
$$

$$
\frac{\partial T}{\partial t}+v \frac{\partial T}{\partial x}=\frac{1}{\epsilon} g(R e|v|)|v|\left(T_{w}(x)-T\right)
$$

for $t>0$, and $x \in(0,1)$

$$
v(0)=v_{0}, \quad T(x, 0)=T_{0}(x)
$$

where for $\xi>0, f(\xi)$ is a continuous function which satisfies (1.7). Here $f, T_{0}$ and $T_{w}$ are periodic given functions with period one (cf. Section 2 below for precise functional assumptions on them), and so will be $T(x, t)$ with respect to the space coordinate $x$. To call attention to this fact, we have replaced the integral symbol in (1.6a) by $\oint$ in $(1.8 a)$ above. This notation will be retained henceforth.

As to the precedings of this work, there is a large literature devoted to the asymptotics of models alike to (1.8), which are usually referred to as thermosyphons. We should first mention the pioneering papers by Keller $[K]$ and Welander $[W]$, and refer to Chen $[C]$, Hart $[H]$, Sen, Ramos and Treviño $[S R T]$ and Liñán $[L]$ (among others) for recent interesting work on this topic. Of course, this bibliographical relation is far from being complete. In particular, further related work can be found in the references included in the previous papers. We should point out that most of these works used formal methods (basically, singular perturbation techniques) in their analysis, and quite often specialized to particular geometries (mainly toroidal) of the circuit considered.

Recently, Herrero and Velázquez considered in $[H V]$ a simplified version of (1.8). Namely, they replaced the right hand side in (1.8) by a prescribed heat flux $q(x)$, and function $g(\operatorname{Re}|v|)$ by a constant (which essentially amounts, to substitute $g$ by its asymptotic value as $\operatorname{Re}|v| \rightarrow \infty)$. Moreover, they dropped the term $\epsilon \frac{d v}{d t}$ in the left hand side of (1.8a). This last assumption was motivated by consideration of the intermediate asymptotics of the model, a stage in which such hypothesis is suggested by formal analysis in $[L] . A$ somewhat surprising result which was proved in $[H V]$ is that stationary solutions of such system are 
generically linearly unstable under small perturbations of the geometry of the pipe or the heating applied there. We refer to $[H V]$ for details and related results.

The paper is concerned with the rigorous analysis of (1.8) for large values of the Reynolds number Re. Our plan here is as follows. Global existence and uniqueness are briefly discussed in a suitable functional frame in Section 2 below. Section 3 is then devoted to the study and characterization of stationary solutions of (1.8) when $R e>>1$. We introduce there a meromorphic function $L(z)$ (cf. (3.8)) which plays a central role in our analysis. In particular, we shall show later in Section 4 that sharp asymptotic estimates on the zeroes of $L(z)$ provide the key to establish a linear stability analysis of the stationary solutions for large Reynolds numbers. Such stability analysis makes the content of Section 4, which is the last in the paper.

Acknowledgements. This work has been partially done while the author was visiting the Institute for Mathematics and its Applications at the University of Minnesota as a Fulbright scholar, during the year 1991-92. It has also been partially supported by CICYT Grant PB90-0235. The author is very grateful to Professors M.A. Herrero and A. Liñán for a number of interesting discussions during the preparation of the manuscript.

\section{GLOBAL EXISTENCE AND UNIQUENESS.}

Consider the following problem

$$
\begin{gathered}
\epsilon \frac{d v}{d t}=\oint T(x, t) f(x) d x-g(\operatorname{Re}|v|)|v| v \text { for } t>0, \\
\frac{\partial T}{\partial t}+v \frac{\partial T}{\partial x}=\frac{1}{\epsilon} g(\operatorname{Re}|v|)|v|\left(T_{w}(x)-T\right)
\end{gathered}
$$

for $t>0$ and $x \in(0,1)$

$$
\begin{aligned}
& v(0)=v_{0}, \quad T(x, 0)=T_{0}(x) \\
& T(0, t)=T(1, t) \text { for any } t>0
\end{aligned}
$$

where $\epsilon>0$ and $R e>0$. Define $H^{1}(0,1)=\left\{h \in L^{2}(0,1): h^{1} \in L^{2}(0,1)\right\}$ and denote by $H_{p}^{1}$ the set consisting on the periodic extension to the whole line of those functions $h \in H^{1}(0,1)$ such that $h(0)=h(1)$. We shall assume that $f, T_{w}$ and $T_{0}$ belong to $H_{p}^{1}$ and $f(x)$ is such that $\int_{0}^{1} f(x) d x=0$. As to $g(\xi)$, in addition to (1.7) we shall require the following 


$$
g(\xi) \geq \eta>0 \text { for any } \xi \in(0, \infty)
$$

$$
\xi g(\xi) \in C^{1} \text { for } \xi>0
$$

These assumptions will be retained henceforth.

We shall say that a pair of functions $(v(t), T(x, t))$ is a solution of (2.1)-(2.4) in a time interval $I=\left[0, \tau_{0}\right)$ if:

i) $v(t) \in C^{1}\left[0, \tau_{0}\right)$,

ii) $T(\cdot, t) \in C^{1}\left(\left[0, \tau_{0}\right): H_{p}^{1}\right)$,

iii) (2.1)-(2.4) are satisfied for $t \in\left[0, \tau_{0}\right)$.

If these conditions hold true for any $\tau_{0}>0$, the solution is said to be global. We now proceed to prove the following result

THEOREM 2.1. Let $f, T_{0}, T_{w}$ and $g$ be given functions satisfying our previous assumptions. Then there exists a unique global solution of (2.1)-(2.4).

Proof. a) Local existence follows from a classical fixed point argument. By standard results, any local solution of (2.1)-(2.4) will satisfy

$$
T(x, t)=\exp \left(-\frac{1}{\epsilon} \int_{0}^{1} g(R e|v(s)|)|v(s)| d s\right) T_{0}\left(x-\int_{0}^{t} v(s) d s\right)+
$$

$+\frac{1}{\epsilon} \int_{0}^{t} \exp \left(-\frac{1}{\epsilon} \int_{s}^{t} g(\operatorname{Re}|v(\lambda)|)|v(\lambda)| d \lambda\right) g(\operatorname{Re}|v(s)|)|v(s)| T_{w}\left(-\int_{s}^{t} v(\lambda) d \lambda\right) d s$,

and

$$
\begin{gathered}
v(t)=v_{0}-\frac{1}{\epsilon} \int_{0}^{t} g(\operatorname{Re}|v(s)|)|v(s)| v(s) d s+ \\
+\frac{1}{\epsilon} \int_{0}^{t} \exp \left(-\frac{1}{\epsilon} \int_{s}^{t} g(\operatorname{Re}|v(\lambda)|)|v(\lambda)| d \lambda\right)\left(\int_{0}^{1} f(x) T^{0} x-\int_{0}^{s} v(\lambda) d \lambda\right) d x d s \\
+\frac{1}{\epsilon^{2}} \int_{0}^{t} d s \int_{0}^{s} \exp \left(-\int_{\lambda}^{s} g(\operatorname{Re}|v(r)|)|v(r)| d r\right) g(\operatorname{Re}|v(\lambda)|)|v(\lambda)| \\
\left(\int_{0}^{1} f(x) T_{w}\left(x-\int_{\lambda}^{s} v(r) d r\right) d x\right) d \lambda \\
\equiv S v(t) .
\end{gathered}
$$


whenever $t$ lies in the existence interval of $(v, T)$. For a given constant $M>0$, we now consider the space $X \equiv X_{\delta, M}=\left\{u \in C[0, \delta]:\left|u(t)-v_{0}\right| \leq M\right.$ for $\left.t \leq \delta\right\}$, endowed with the supremum norm. A straightforward computation reveals then that, for $\delta>0$ small enough, $S$ is a contractive operator from $X$ into itself. This yields the existence of a unique solution $(v, T)$ of (2.1)-(2.4) for small times.

b) To obtain global existence we recall that, by our assumptions on $g$, there exist positive constants $\sigma, A$ and $B$ such that

$$
\frac{\sigma}{R e} \leq g(\operatorname{Re}|v(t)|)|v(t)| \leq A+B|v(t)|
$$

as for as $v(t)$ is well defined. For any $h \in L^{2}$, let us denote by $\|h\|$ the $L^{2}$-norm of $h$. Substituting (2.6) into (2.1) and multiplying both sides there by (sgnv), we obtain

$$
\begin{gathered}
\epsilon \frac{d|v|}{d t} \leq-g(\operatorname{Re}|v|)|v|^{2}+\|f\|\left\|T_{0}\right\| \\
+\|f\|\left\|T_{w}\right\|\left(\frac{1}{\epsilon} \int_{0}^{t} e^{-\frac{\sigma(t-s)}{R e}} g(\operatorname{Re}|v(s)|)|v(s)| d s\right)
\end{gathered}
$$

and, by (2.5a)

$$
\begin{gathered}
\epsilon \frac{d|v|}{d t} \leq \eta^{2}|v|^{2}+\|f\|\left\|T_{0}\right\|+\frac{\|f\|\left\|T_{w}\right\|}{\epsilon R e} \int_{0}^{t} e^{-\frac{\sigma(t-s)}{R e}}(A+\operatorname{BRe}|v(s)|) d s \\
\leq \eta^{2}|v|^{2}+\|f\|\left\|T_{0}\right\|+\|f\|\left\|T_{w}\right\| \frac{A}{\sigma \epsilon}+\frac{B}{\epsilon} \int_{0}^{t} e^{-\frac{\sigma(t-s)}{R e}}|v(s)| d s
\end{gathered}
$$

Suppose now that $|v(s)| \leq C$ for $s \in[0, t]$, where $C>2\left|v_{0}\right|$ will be precised later. Then (2.8) yields

$$
\epsilon \frac{d|v|}{d t} \leq \eta^{2}|v|^{2}+\|f\|\left\|T_{0}\right\|+\|f\|\left\|T_{w}\right\| \frac{A}{\sigma \epsilon}+\frac{\mathrm{BRe}}{\sigma \epsilon}
$$

In particular, if $|v(t)|=C$, we would have $\frac{d|v(t)|}{d t}<0$ provided that $C$ is selected large enough. We have obtained a bound for $|v(t)|$ which is independent of its existence interval. Using (2.6), we obtain a similar bound for $T(x, t)$, and we now conclude by means of a standard continuation argument.

\section{STATIONARY SOLUTIONS FOR LARGE REYNOLDS NUMBERS.}

In this Section we shall analyze the set of stationary solutions of (2.1)-(2.4) in the case where $R e>>1$. We will show that, according to their velocity, there can be classified as fast solutions (when $|v| \approx 1$ ) and slow solutions, which are such that $|v| \approx \frac{1}{R e}$. 
To begin with, stationary solutions satisfy

$$
\begin{gathered}
\oint f(x) T(x) d x=g(\operatorname{Re}|v|)|v| v \\
v \frac{\partial T}{\partial x}=\frac{1}{\epsilon} g(\operatorname{Re}|v|)|v|\left(T_{w}-T\right)
\end{gathered}
$$

$$
T(0)=T(1)
$$

where $f, T_{w}, \epsilon$ and $f$ are as in the previous Section. We shall often use Fourier expansions for $f, T_{w}$ and $T$, which will be written as follows

$$
f(x)=\sum_{-\infty}^{\infty} a_{k} e^{2 \pi i k x} \text { where } a_{0}=0\left(\text { since } \int_{0}^{1} f(x) d x=0\right)
$$

$$
T_{w}(x)=\sum_{-\infty}^{\infty} b_{k} e^{2 \pi \mathrm{ikx}}
$$

$$
T(x)=\sum_{-\infty}^{\infty} c_{k} e^{2 \pi \mathrm{ikx}}
$$

Furthermore, since all functions considered are real, one has that $a_{k}=\bar{a}_{-k}$ for any $k$, and a similar result holds for $b_{k}, c_{k}$. Clearly, one has that

$$
\begin{aligned}
\|f\|_{H^{1}}^{2} & =\sum_{-\infty}^{\infty}\left(1+k^{2}\right) a_{k}^{2}<+\infty \\
\left\|T_{w}\right\|_{H^{1}}^{2} & =\sum_{-\infty}^{\infty}\left(1+k^{2}\right) b_{k}^{2}<+\infty
\end{aligned}
$$

The following quantity will play an important role in what follows

$$
\chi_{0}=\int_{0}^{1} f(x) T_{w}(x) d x=\sum_{-\infty}^{\infty} a_{k} \bar{b}_{k}
$$


From (3.1) and (3.4), we obtain

$$
c_{k}=\frac{|v|}{\epsilon} g(\operatorname{Re}|v|) b_{k}\left(\frac{1}{\epsilon} g(\operatorname{Re}|v|)|v|+2 \pi k i v\right)^{-1}
$$

for any integer $k,-\infty<k<+\infty$

Taking into account (3.7), it turns out that (3.1) can be recast as follows

$$
\epsilon v^{2}=\sum_{-\infty}^{\infty} \frac{a_{k} \bar{b}_{k}}{z-2 \pi k_{i}} \equiv L(z)
$$

where

$$
z=\frac{1}{\epsilon} \operatorname{sgn}(v) g(R e|v|)
$$

Analysis of function $L(z)$ in $(3.8 a)$ will be instrumental in deriving the main results in this Section (cf. Theorem 3.2 below). One readily sees that $L(z)$ is a meromorphic function with poles at points $\tilde{z}_{j}=2 \pi i j$ for which $\overline{a_{j} b_{j}} \neq 0$. To avoid a separate study of the cases where $\overline{a_{j} b_{j}}=0$, we now introduce some definitions. We shall say that $L(z)$ has generalized poles at points $\tilde{z}_{j}=2 \pi i j$ for $j= \pm 1, \pm 2, \ldots A$ point $z_{0}$ will be said to be a generalized zero of $L(z)$ if either $z_{0}$ is a zero of $L(z)$ or $z_{0}=2 \pi i j$ and $a_{j} \bar{b}_{j}=0$ for some $j$. Notice that this last case can be viewed as a collapse of a zero and a pole, which takes place when $a_{j} \bar{b}_{j}=0$. It is readily seen that the classical arguments principle in complex variable as well as Rouche's Theorem (which follows from it) still hold true when zeroes and poles are replaced by generalized zeroes and generalized poles respectively. We shall keep to this terminology from now on, although the term generalized will be dropped for convenience. We thus shall refer henceforth to zeroes and poles without any further specification.

Some useful properties of function $L(z)$ are gathered in the following

Lemma 3.1. Assume that $\chi_{0} \neq 0$ in (3.6). Then the zeroes of $L(z)$ can be labelled as a sequence $\left\{z_{j}\right\}: j=0, \pm 1, \pm 2, \ldots$. Moreover, there holds

$$
\lim _{|j| \rightarrow \infty}\left|z_{j}-2 \pi i j\right|=0
$$

Remark. It follows from (3.9) that $L(z)$ has at most a finite number of real zeroes.

Proof of Lemma 3.1. It will follow from a suitable application of the argument principle, a technique which will be repeatedly used in the sequel. Consider the contour 
$\gamma_{N}=\left\{z \quad \in \mathbb{C}: z=(2 N+1) \pi e^{i \theta}\right.$ with $\left.\theta \in[0,2 \pi]\right\}$, where $N=0,1,2, \ldots$ For any $z \in \gamma_{N}$, we have that

$$
\begin{gathered}
\left|L(z)-\frac{\chi_{0}}{z}\right| \leq \sum_{|k|>\frac{N}{2}} \frac{2 \pi|k|\left|a_{k} \bar{b}_{k}\right|}{|z||z-2 \pi i k|}+\sum_{|k| \leq \frac{N}{2}} \frac{2 \pi|k|\left|a_{k} \bar{b}_{k}\right|}{|z||z-2 \pi i k|} \\
\equiv S_{1}+S_{2}
\end{gathered}
$$

Since $|z-2 \pi i k| \geq \pi$ whenever $z \in \gamma_{N}$, it follows from (3.5) that

$$
\begin{gathered}
S_{1} \leq \frac{2}{|z|}\left(\sum_{|k|>\frac{N}{2}} k^{2}\left|a_{k}\right|^{2}\right)^{1 / 2}\left(\sum_{|k|>\frac{N}{2}} k^{2}\left|b_{k}\right|^{2}\right)^{1 / 2} \\
\equiv o\left(\frac{1}{|z|}\right) \text { as } N \rightarrow \infty
\end{gathered}
$$

If $|k| \leq \frac{N}{2}$, then $|z-2 \pi i k| \geq|z|-2 \pi|k| \geq \pi N$ whenever $z \in \gamma_{N}$, whence

$$
S_{2} \leq \frac{2}{N|z|}\|f\|_{H^{1}}\left\|T_{w}\right\|_{H^{1}}=o\left(\frac{1}{|z|}\right) \text { as } N \rightarrow \infty
$$

for $z \in \gamma_{N}$. We thus have that

$$
\begin{gathered}
\left|L(z)-\frac{\chi_{0}}{z}\right|=o\left(\frac{1}{|z|}\right) \text { whenever }|z|=(2 N+1) \pi \\
\text { and } N \rightarrow \infty
\end{gathered}
$$

It then follows from the argument principle that the number of zeroes of $L(z)$ exceeds in one to that to their poles at the interior of $\gamma_{N}$ when $N>>1$. Recalling (3.3a), we conclude that zeroes of $L(z)$ can be labelled as $z_{0}, z_{ \pm 1}, z_{ \pm 2}, \ldots$ Moreover, for large enough $N$, there are exactly two such zeroes in the annulus $A_{N}=\{z:(2 N+1) \pi<|z|<(2 N+3) \pi\}$. We now proceed to derive (3.9). To this end, we notice that

$$
\left|L(z)-\frac{a_{j} \bar{b}_{j}}{z-2 \pi i j}-\frac{\chi_{0}}{z}\right|=o\left(\frac{1}{|z|}\right) \text { as }|j| \rightarrow \infty
$$

uniformly on sets $|z-2 \pi i j| \leq \frac{1}{10}$. 
The proof of (3.11) is similar to that of (3.10) and will therefore be omitted. Consider now the contours $C_{j}=\left\{z \in \mathbb{C}:|z-2 \pi i j|=\frac{4 \pi|j| a_{j} \bar{b}_{j}}{\left|\chi_{0}\right|}\right\}$. Recalling (3.5), one obtains after a routine computation that

$$
\left|\frac{a_{j} \bar{b}_{j}}{z-2 \pi i j}+\frac{\chi_{0}}{z}\right| \geq \frac{\left|\chi_{0}\right|}{8|z|} \text { for } z \in C_{j},|j|>>1
$$

From (3.11), (3.12) and the argument principle, we deduce that $L(z)$ and $L_{1}(z)=\left(\frac{a_{j} \bar{b}_{j}}{z-2 \pi i j}+\frac{\chi_{0}}{z}\right)$ have the same number of zeroes within the $\operatorname{discs} B_{j}=\left\{z:|z-2 \pi i j|<\frac{\left.4 \pi|j| \mid a_{j} \bar{b}_{j}\right\rfloor}{\left|\chi_{0}\right|}\right\}$. We now conclude by observing that zeroes of $L_{1}(z)$ are located at points

$$
\tilde{z}_{j}=(2 \pi i j)\left(1+\frac{a_{j} \bar{b}_{j}}{\chi_{0}}\right)^{-1}
$$

so that, by Taylors expansion

$$
\left|\tilde{z}_{j}-2 \pi i j\right|<4 \pi|j|\left|a_{j} \bar{b}_{j}\right|\left|\chi_{0}\right|^{-1} \text { if }|j|>>1,
$$

and recalling (3.5), (3.9) follows.

We now impose further conditions on the function $g(\xi)$, namely

There exists $\chi_{0}$ and $\xi_{1}$ with $0<\xi_{0}<\xi_{1}<+\infty$ such that

$$
\begin{gathered}
g^{\prime}(\xi)<0 \text { for } 0<\xi<\xi_{0}, g^{\prime}(\xi)>0 \text { for } \xi_{0}<\xi<\xi_{1} \text { and } \\
g^{\prime}(\xi)<0 \text { for } \xi>\xi_{1} \text {. At } \xi_{0}, \xi_{1} \text { we have that } g\left(\xi_{1}\right)=M, g\left(\xi_{2}\right)=m .
\end{gathered}
$$

$$
\text { There exist } B>0 \text { such that }
$$

$$
\begin{aligned}
& g(\xi)=1+\frac{B}{\xi}+o\left(\frac{1}{\xi}\right) \text { as } \xi \rightarrow \infty \\
& g^{\prime}(\xi)=-\frac{B}{\xi^{2}}+o\left(\frac{1}{\xi^{2}}\right) \text { as } \xi \rightarrow \infty
\end{aligned}
$$

In view of (3.13a), it is clear that if $z$ and $v$ are related by (3.8b), we should have $|z| \geq \frac{m}{\epsilon}$. Consider now the set 


$$
S=\left\{z \in \mathbb{C}: L(z)=0 \text { and }|z| \geq \frac{m}{\epsilon}\right\} .
$$

For any $z_{j} \in S$, we define a set of complex numbers $\left\{\xi_{j}^{\ell}\right\}, \ell=1, \ldots, j\left(z_{j}\right)$ given by the roots of the equation

$$
z_{j}=\frac{1}{\epsilon} \operatorname{sgn}(\xi) g\left(\xi_{j}^{\ell}\right)
$$

The main result of this Section is

Theorem 3.2. Assume that hypotheses (3.13) hold, and

$$
\chi_{0} \neq 0, L\left( \pm \frac{1}{\epsilon}\right) \neq 0, L\left( \pm \frac{m}{\epsilon}\right) \neq 0, L\left( \pm \frac{M}{\epsilon}\right) \neq 0 .
$$

Then for large enough Reynolds numbers there exists a one-to-one correspondence between the possible values of the velocity for solutions of (3.1)-(3.3), and the elements of the following set

$$
\begin{aligned}
& I=\left\{\left\{\xi_{j}^{\ell}\right\}: \ell=1, \ldots, j\left(z_{j}\right), \text { where } \xi_{j}^{\ell} \text { and } z_{j}\right. \text { are } \\
& \text { related by (3.14) }\} \bigcup\left\{z: z= \pm \frac{1}{\epsilon} \text { and } L(z)>0\right\} .
\end{aligned}
$$

Moreover, the dependence of the velocity values on Reynolds numbers in such case is described by the following formulae

$$
\begin{gathered}
v_{j}^{\ell}=\frac{\xi_{j}^{\ell}}{R e}+o\left(\frac{1}{R e}\right) \text { as } R e \rightarrow \infty \\
\text { for } \ell=1, \ldots, j\left(z_{j}\right) \\
v_{+}=\left(\frac{1}{\epsilon} L\left\{\frac{1}{\epsilon}\right\}\right)^{1 / 2}+o(1) \text { as } \operatorname{Re} \rightarrow \infty \\
\text { if } L\left(\frac{1}{\epsilon}\right)>0
\end{gathered}
$$




$$
\begin{gathered}
v_{-}=-\left(\frac{1}{\epsilon} L\left\{-\frac{1}{\epsilon}\right\}\right)^{1 / 2}+o(1) \text { as } R e \rightarrow \infty \\
\text { if } L\left(-\frac{1}{\epsilon}\right)>0 .
\end{gathered}
$$

Finally, solutions satisfying (3.16) actually exist.

Remark. Theorem 3.2 states that, under suitable transversality conditions, stationary solutions corresponding to large Reynolds numbers may be of two types: slow solutions, for which velocities are of order $\frac{1}{R e}$, and fast solutions, for which velocities are of order unity. Notice that assumptions (3.14), (3.15) are generic, in the sense of being preserved under small perturbations of $f, T_{w}, \epsilon$ and $\mathrm{g}$.

Proof of Theorem 3.2. We shall consider separately equation (3.8a) in the cases where $|v| \geq(R e)^{-1 / 2}$ and $|v|$ that $|v| \geq(R e)^{-1 / 2}$. Then $g(R e|v|)=1+o(1)$, uniformly as $R e \rightarrow \infty$. This in turns implies that

$$
z=\frac{\operatorname{sgn}(v)}{\epsilon}+o(1), L(z)=L\left(\frac{\operatorname{sgnv}}{\epsilon}\right)+o(1)
$$

uniformly as $R e \rightarrow \infty$ where $z$ and $v$ are related through (3.8b). Since $L\left( \pm \frac{1}{\epsilon}\right) \neq 0$ by assumption, we deduce that for such solutions to exist it is necessary to have $L\left(\frac{1}{\epsilon}\right)>0$ or $L\left(-\frac{1}{\epsilon}\right)>0$, in which cases $(3.17 \mathrm{a})$ or $(3.17 \mathrm{~b})$ respectively hold. To show uniqueness of such solutions, we argue as follows. Let us define

$$
H(v)=L\left(\frac{1}{\epsilon} \operatorname{sgn}(v)\right) g(R e|v|)
$$

Recalling condition (3.13b), we see that for $|v| \geq(R e)^{-1 / 2}$ and large enough $(R e)$

$$
\left|H^{\prime}(v)\right| \leq C(R e)^{-1 / 2}
$$

where here and henceforth $C$ will denote a generic constant, depending possibly on $\|f\|,\left\|T_{w}\right\|$, $\epsilon$ and $g$. Suppose now that there exist two different solutions with velocity values $v_{1}$ and $v_{2}$, and that (3.17a) holds. Then $v_{i} \geq \mu>0(i=1,2)$ for some $\mu$ and $R e>>1$. By (3.8a), one then has

$$
\frac{H\left(v_{1}\right)-H\left(v_{2}\right)}{v_{1}-v_{2}}=\epsilon\left(v_{1}+v_{2}\right) \geq 2 \mu \epsilon
$$

whereas on the other hand, by (3.19) 


$$
\frac{H\left(v_{1}\right)-H\left(v_{2}\right)}{v_{1}-v_{2}}=\frac{1}{v_{1}-v_{2}} \int_{v_{2}}^{v_{1}} H^{\prime}(s) d s \leq C(R e)^{-1 / 2}
$$

From (3.20) one derives a contradiction when $R e>>1$. The case where (3.17b) holds is similar.

Assume now that $|v|<(R e)^{-1 / 2}$. By (3.18) and (3.8a), it then follows that $|H(v)| \leq \epsilon(R e)^{-1 / 2}$. If $R e>>1$, this can only happen if one of the following cases holds

$$
\frac{1}{\epsilon} \operatorname{sgn}(v) g(\operatorname{Re}|v|) \approx z_{j}, \text { where } z_{j} \in S(\text { cf. (3.13a)) }
$$

or

$$
R e|v| \rightarrow 0 \text { as } R e \rightarrow \infty
$$

We next proceed to rule out the second alternative above. Indeed, if (3.21b) occurs, by $(3.13 \mathrm{~b})$, we should have

$$
\begin{gathered}
\frac{A}{2 \xi} \leq g(\xi) \leq \frac{3 A}{2 \xi} \text { if } \xi \text { is small enough } \\
\text { (independently of } R e \text { ) }
\end{gathered}
$$

so that, by $(3.8 \mathrm{~b})$

$$
\frac{A}{2 \epsilon \operatorname{Re}|v|} \leq|z| \leq \frac{3 A}{2 \epsilon \operatorname{Re}|v|} \text { for } R e>>1
$$

We then can use (3.10) and (3.13b), to obtain that for $R e>>1$,

$$
\epsilon v^{2}=|L(z)| \geq \frac{\epsilon\left|\chi_{0}\right|}{3 A} R e|v|
$$

whence

$$
v=0
$$

or 


$$
|v| \geq \frac{\left|\chi_{0}\right| R e}{3 A}
$$

If (3.22b) holds, we get at once a contradiction with the assumption $|v| \leq(R e)^{-1 / 2}$. If on the other hand $v=0$, we deduce from (3.2) that $T(x) \equiv T_{w}(x)$, in which case (3.1) yields $\chi_{0}=0$, which contradicts (3.14). Then (3.21a) must be satisfied. Taking into account $(3.13 \mathrm{~b})$, it follows that such solutions (if any) should verify (3.16). It remains yet to prove existence and uniqueness for this last type of solutions. To this end, we use the fact that

$$
L\left( \pm \frac{m}{\epsilon}\right) \neq 0, L\left( \pm \frac{M}{\epsilon}\right) \neq 0, L\left( \pm \frac{1}{\epsilon}\right) \neq 0 .
$$

(cf. (3.15a)). Together wit (3.15b), this implies that $g^{\prime}\left(\xi_{j}^{\ell}\right) \neq 0$ for any $j$ and $\ell$ as before. Using Taylor's expansion, we then obtain that

$$
\begin{gathered}
H(v)=\frac{R e \operatorname{sgn}(v)}{\epsilon}\left[L^{\prime}\left(\frac{\operatorname{sgn}(v) g\left(\xi_{j}^{\ell}\right)}{\epsilon}\right) g^{\prime}\left(\xi^{\ell_{j}}\right)\left(v-\frac{\xi_{j}^{\ell}}{R e}\right)+o\left(v-\frac{\xi_{j}^{\ell}}{R e}\right)\right] \\
\text { for } v-\frac{\xi_{j}^{\ell}}{R e}=o\left(\frac{1}{R e}\right), \text { uniformly as } R e \rightarrow \infty .
\end{gathered}
$$

It is then readily seen that, for $\chi>0$ small enough, $H\left(\frac{\xi_{j}^{\ell}}{R e}-\frac{\delta}{R e}\right)$ and $H\left(\frac{\xi_{j}^{\ell}}{R e}+\frac{\delta}{R e}\right)$ have opposite signs, whence existence follows. Uniqueness is obtained by noting that

$$
\frac{d}{d r}\left(H(r)-\epsilon r^{2}\right)=\frac{R e \operatorname{sgn}(r)}{\epsilon} L^{\prime}\left(\frac{\operatorname{sgn}(r) g(\operatorname{Re}|r|)}{\epsilon}\right)-2 \epsilon r
$$

so that $\frac{d}{d v}\left(H(v)-\epsilon v^{2}\right)$ has a definite sign for $v \in\left(\frac{\xi_{j}^{\prime}}{R e}-\frac{\delta}{R e}, \frac{\xi_{j}^{\ell}}{R e}+\frac{\delta}{R e}\right)$ when $\delta>0$ is small enough.

\section{LINEAR STABILITY OF STATIONARY SOLUTIONS FOR LARGE REYNOLDS NUMBERS.}

\subsection{Preliminaries. Linear stability of fast solutions.}

In this Section we shall perform a linear stability analysis of the stationary solutions considered in Section 3. To this end, it will be useful to introduce a new velocity related variable $\Phi$ and a new time $s$, defined as follows 


$$
\Phi=\frac{|v| v}{2}
$$

$$
d s=\frac{1}{\epsilon} g(\operatorname{Re}|v|)|v| d t
$$

It is then readily seen that $(2.1),(2.2)$ can be rewritten in the form

$$
\frac{d \Phi}{d s}+2 \Phi=\frac{1}{g(\operatorname{Re}|v|)} \oint f(x) T(x, s) d s,
$$

$$
\frac{\partial T}{\partial s}+\frac{\epsilon \operatorname{sgn} \Phi}{g(\operatorname{Re}|v|)} \cdot \frac{\partial T}{\partial x}=\left(T_{w}(x)-T(x, s)\right)
$$

Classical linear stability theory proceeds by setting

$$
\begin{gathered}
\Phi=\Phi_{s}+\delta \Phi=\Phi_{s}+c e^{-\lambda s}+\ldots, \\
T=T_{s}+\delta T=T_{s}+e^{-\lambda s} \psi+\ldots,
\end{gathered}
$$

where it is assumed that $\delta \Phi<<1, \delta T<<1$, as $s \rightarrow \infty, \Phi_{s}$ and $T_{s}$ being stationary solutions of (4.2), (4.3). Retaining only first order terms, standard computations yield then

$$
\begin{gathered}
(2-\lambda) c=-Q \int_{0}^{1} f(x) T_{s}(x) d x+\left(g\left(\operatorname{Re}\left|2 \Phi_{s}\right|\right)^{1 / 2}\right)^{-1} \int_{0}^{1} f(x) \psi(x) d x \\
-\lambda \psi+\frac{\psi^{\prime}}{2}-\epsilon c \operatorname{sgn} \Phi_{s} Q T_{s}=-\psi
\end{gathered}
$$

where

$$
Q=\frac{g^{\prime}\left(\operatorname{Re}\left|2 \Phi_{s}\right|^{1 / 2}\right) R e \operatorname{sgn}\left(\Phi_{s}\right)}{g\left(\operatorname{Re}\left|2 \Phi_{s}\right|^{1 / 2}\right)\left(2\left|\Phi_{s}\right|\right)^{1 / 2}}
$$

and $z$ is given in $(3.8 b)$. If we now write 


$$
\psi(x)=\sum_{\infty}^{\infty} d_{k} e^{2 \pi \mathrm{ikx}}
$$

Then, recalling (3.4) and (3.7), we see that (4.4) can be written in the form

$$
\begin{gathered}
(2-\lambda) c=-Q\left(\sum \bar{a}_{k} c_{k}\right) c+g\left(\operatorname{Re}\left|2 \Phi_{s}\right|^{1 / 2}\right)^{-1} \sum \bar{a}_{k} d_{k} \\
\left((1-\lambda)+\frac{2 \pi i k}{z}\right) d_{k}=c Q \epsilon \operatorname{sgn}\left(v_{s}\right) 2 \pi \mathrm{ikc} c_{k}: k= \pm 1, \pm 2, \ldots
\end{gathered}
$$

Set now

$$
\mu=(1-\lambda) z
$$

Then straightforward (but tedious) calculations show that there exists nontrivial solutions of (4.6) if and only if

$$
\begin{gathered}
\mu \neq 2 \pi k i: \text { and } S(\mu)=1+\frac{\mu}{z}+\frac{Q \mu z}{\mu-z} L(z)-L(\mu)= \\
v_{s}^{2}-L(\mu)=0,
\end{gathered}
$$

or $\mu=2 \pi k i$ and $a_{k} \bar{b}_{k}=0$, where $k= \pm 1, \pm 2, \ldots, v_{s}$ is a stationary velocity value (related to $\Phi_{s}$ via (4.1b)), and $Q, L$ are respectively given in (4.4c) and (3.8a). Notice that without loss of generality, the eigenvalues $\mu$ in (4.7) are given by the roots of

$$
\left(1-e^{\mu}\right) S(\mu)=0
$$

Consider now $S$ as a function of the complex variable $\mu$. Then the poles of $S(\mu)$ are located at $\mu=2 \pi i k, k= \pm 1, \pm 2, \ldots$ In particular, $\mu=z$ is not a pole for the meromorphic function S. By means of a slight modification of the arguments leading to the proof of Lemma 3.1 , we then see that

$$
S(\mu) \approx \frac{\mu}{z} \text { as } N \rightarrow \infty \text { if }|\mu|=(2 N+1) \pi
$$

and 
The set of generalized zeroes of $S(\mu)$ can be labelled

$$
\text { as a sequence }\left\{\mu_{j}\right\} \text { where } j=0, \pm 1, \pm 2, \pm 3, . .
$$

Furthermore

$$
(2|j|-1) \pi<\left|\mu_{j}\right|<(2|j|+1) \pi \text { for } j= \pm 1, \pm 2, \pm 3, \ldots
$$

We next set out to estimate the roots of $\left(1-e^{\mu}\right) S(\mu)$. As a first result, we shall prove:

Lemma 4.1. Assume that the hypotheses in Theorem 3.2 are satisfied and $v_{s}$ behaves as in (3.17). Then there holds

$$
|Q|=O\left(\frac{1}{R e}\right) \text { as } R e \rightarrow \infty
$$

Moreover, there exists a constant $C$ independent of Re and $j$, such that

$$
\text { There is one root } \mu_{0} \text { of } S(\mu) \text { satisfying }
$$

$$
\left|\mu_{0}+z\right| \leq C|Q| \text { as } R e \rightarrow \infty
$$

$$
\begin{gathered}
\text { For } j= \pm 1, \pm 2, \pm 3, \ldots \text { there is a unique root of }\left(1-e^{\mu}\right) S(\mu) \text { satisfying } \\
\qquad\left|\mu_{j}-2 \pi i j\right| \leq \frac{C|Q|\left|\bar{a}_{j} b_{j}\right|}{|j|} \text { as } R e \rightarrow \infty
\end{gathered}
$$

Proof. Estimate (4.11) follows at once from (4.4c) and (3.13b). Furthermore, under our current assumptions $z \rightarrow \pm \frac{1}{\epsilon}$ as $R e \rightarrow \infty$. If we now fix $\delta>0$, we have that

$$
\left|S(\mu)-\left(1+\frac{\mu}{2}\right)\right|=\left|Q \mu z \frac{L(z)-L(\mu)}{z-\mu}\right| \leq K|Q|
$$

for some $K>0$, provided that $|\mu+z| \leq \delta$.

Set now $|\mu+z|=2 K|z||Q|$. By (4.11), we see that $|\mu+z| \leq \delta$ if $R e>>1$. One then has that

$$
\left|S(\mu)-\left(1+\frac{\mu}{2}\right)\right| \leq K|Q|<2 K|Q|=\left|1+\frac{\mu}{z}\right|
$$


Then by Rouche's Theorem we obtain the existence of a root of $S(\mu)$ at the interior of the ball $B_{1}=(\mu:|\mu+z|<2 K|Q \| z|)$ if $R e>>1$, whence (4.12a) follows. As to (4.12b) it suffices to consider the case $a_{j} \bar{b}_{j} \neq 0$, since otherwise the result holds in view of (4.8) and (4.9). A first estimate on the location of the zeroes $\left\{\mu_{j}\right\}$ is then provided by (4.10). Assume now that

$$
|\mu-2 \pi i j|=\frac{2|Q||j| z^{2}\left|a_{j} \bar{b}_{j}\right|}{|2 \pi i j-z||2 \pi i j+z|}
$$

Standard calculations yield then

$$
\begin{gathered}
\left|S(\mu)-1+\frac{2 \pi i j}{z}+\frac{Q \mu z}{2 \pi i j-z} \cdot \frac{a_{j} \bar{b}_{j}}{\mu-2 \pi i j}\right| \\
\leq \frac{|\mu-2 \pi i j|}{|z|}+\frac{|Q||\mu||z|}{|\mu-z|}\left(|L(z)|+\left|\sum_{k \neq j} \frac{a_{k} \bar{b}_{k}}{\mu-2 \pi i k}\right|\right) \\
\leq C|Q|
\end{gathered}
$$

where $C$ depends on $\|f\|,\left\|T_{w}\right\|$ and $z$, but not on $|j|$. In a similar way, we obtain that, if (4.13) holds

$$
\left|1+\frac{2 \pi i j}{z}-\frac{Q \mu z}{2 \pi i j-z} \cdot \frac{a_{j} \bar{b}_{j}}{\mu-2 \pi i j}\right| \geq \frac{1}{2}\left|1+\frac{2 \pi i j}{z}\right|
$$

Taking into account (4.14), the conclusion follows by Rouche's Theorem, since there are only a finite number of such roots in $|j|$ is bounded.

An immediate consequence of Lemma 4.1, is the following

Corollary 4.2. Assume that the hypotheses in Theorem 3.2 hold. Then stationary solutions whose velocities satisfy (3.17) are linearly stable for large Reynolds numbers.

Proof. Recalling (4.7) and (4.12), we readily see that as $R e \rightarrow \infty$, the eigenvalues of the linearized problem satisfy

$$
\lambda_{0}=2+O\left(\frac{1}{R e}\right)
$$




$$
\left|\lambda_{k}-1+\frac{2 \pi i k}{z}\right| \leq \frac{C}{R e} \cdot \frac{\left|a_{k} \bar{b}_{k}\right|}{|k|} \text { for } k= \pm 1, \pm 2, \ldots
$$

where $C$ depends on $z, f$ and $T_{w}$.

\subsection{Linear stability of slow solutions. Statement of the main result. Re- fined estimates on $\mathrm{L}(\mathrm{z})$.}

We shall now consider the case where (3.16) holds. Then the function $Q$ given in (4.4c) is such that

$$
Q=\frac{g^{\prime}\left(\operatorname{Re}\left|v_{s}\right|\right) R e}{\left(g\left(\operatorname{Re}\left|v_{s}\right|\right)\right)^{2}} \sim \frac{g^{\prime}\left(\xi_{j}^{\ell}\right) R e^{2}}{g\left(\xi_{j}^{\ell}\right)^{2} \xi_{j}^{\ell}} \text { as } R e \rightarrow \infty
$$

where $v_{s}$ and $\xi_{j}^{\ell}$ are related as explained in the statement of Theorem 3.2. Notice that $g^{\prime}\left(\xi_{j}^{\ell}\right) \neq 0$ since $L\left( \pm \frac{m}{\epsilon}\right) \neq 0, L\left( \pm \frac{1}{\epsilon}\right) \neq 0$ and $L\left( \pm \frac{M}{\epsilon}\right) \neq 0$. In what follows, we shall require that

$$
\frac{M_{0}}{\chi_{0}}+\frac{g\left(\xi_{j}^{\ell}\right)^{2}}{g^{\prime}\left(\xi_{j}^{\ell}\right) \xi_{j}^{\ell} z_{j}^{2}}+\epsilon \neq 0, \text { where }
$$

$M_{0}=\sum_{-\infty}^{\infty} 2 \pi i k a_{k} \bar{b}_{k}, \chi_{0}$ is given in (3.6), $z_{j}$ is one of the zeroes of $L(z)$ and $z_{j}, \xi_{j}^{\ell}$ are related through (3.14b) (cf. also (3.16)).

Let us consider now a stationary solution whose velocity $v \equiv v_{s}$ satisfies (3.16). Our goal consists in proving.

Theorem 4.3. Assume that the hypotheses in Theorem 3.2 are fulfilled. Suppose also that (3.16) and (4.17) hold. Then the roots of $\left(1-e^{\mu}\right) S(\mu)$ can be labelled as a sequence $\left\{\mu_{k}\right\}: k=0, \pm 2, \pm 3, \ldots$ whose elements have the following asymptotic behaviours.

$$
\begin{gathered}
\left|\mu_{k}-z_{k}\right|=o(1) \text { as } R e \rightarrow \infty \text { for } k= \pm 2, \pm 3, . . \\
\text { and } k \neq j, \\
\mu_{0}=o(1) \text { as } \operatorname{Re} \rightarrow \infty \\
\mu_{ \pm 1}= \pm\left(Q z^{2} \chi_{0}\right)^{1 / 2}+\frac{q \epsilon z^{2}\left(\xi_{j}^{\ell}\right)^{2}}{2}+\frac{M_{0}}{2 \chi_{0}}+o(1), \\
\text { as } \operatorname{Re} \rightarrow \infty, \text { where } q=\lim _{R e \rightarrow \infty} \frac{Q}{(\operatorname{Re})^{2}}(\text { cf. (4.16)) }
\end{gathered}
$$


Remark. Notice that all the assumptions in Theorem 4.3 are generic in the sense of being preserved under small perturbations on $f, T_{w}, g$ and $\epsilon$. Notice also that $\mu_{k}=2 \pi i k$ is one root of $\left(1-\epsilon^{\mu}\right) S(\mu)$ if $a_{k} \bar{b}_{k}=0$.

For convenience, the proof of Theorem 4.3 will be divided into several steps. We first need a number of refined estimates on $L(z)$, and its sequence of zeroes $\left\{z_{k}\right\}$. We begin by

LEMMA 4.4. Under the assumptions of Lemma 3.1, there holds

$$
\lim _{|k| \rightarrow \infty} \frac{\left|z_{k}-2 \pi i k\left(1-a_{k} \bar{b}_{k} \chi_{0}^{-1}\right)\right|}{|k|\left|a_{k} \bar{b}_{k}\right|}=0
$$

Proof. It follows from a suitable application of Rouche's Theorem. Take $\sigma>0$ fixed, and consider the disks

$$
B_{j}=\left\{z \in \mathbb{C}: z=2 \pi i k\left(1-\frac{a_{k} \bar{b}_{k}}{\chi_{0}}\right)+\sigma\left|k \| a_{k} \bar{b}_{k}\right| e^{i \theta}, \theta \in[0,2 \pi]\right\}
$$

Since for $\delta>0$ small enough we have that $(1+\delta)^{-1}=1-\delta+O\left(\delta^{2}\right)$, standard calculations show that, if $|k|>>1$ and $\sigma>0$ is sufficiently small,

$$
\begin{gathered}
\left|\frac{\chi_{0}}{z}+\frac{a_{k} \bar{b}_{k}}{z-2 \pi i k}\right|= \\
=\mid \frac{\chi_{0}}{2 \pi i k}\left(1+\frac{a_{k} \bar{b}_{k}}{\chi_{0}}-\frac{\sigma}{2 \pi i} \frac{\left|a_{k} \bar{b}_{k}\right| k \mid e^{i \theta}}{k}+O\left|a_{k} \bar{b}_{k}\right|^{2}\right)- \\
-\frac{\chi_{0}}{2 \pi i k}\left(1-\frac{i \sigma \chi_{0}}{2 \pi} \cdot \frac{\left|a_{k} \bar{b}_{k}\right| k \mid e^{i \theta}}{k a_{k} \bar{b}_{k}}+O\left(\sigma^{2}\right)\right) \\
\geq \frac{\sigma \chi_{0}^{2}}{8 \pi^{2}|k|}
\end{gathered}
$$

Putting together (4.22) and (3.11), we deduce that $L(z)$ and $\left(\frac{\chi_{0}}{z}+\frac{a_{k} \bar{b}_{k}}{z-2 \pi i k}\right)$ have the same number of zeroes at the interior of $B_{k}$, whence

$$
\left|z_{k}-2 \pi i k\left(1-\frac{a_{k} \bar{b}_{k}}{\chi_{0}}\right)\right|<\sigma|k|\left|a_{k} \bar{b}_{k}\right|
$$

and since $\sigma>0$ can be taken arbitrarily small, (4.21) follows.

We next show

Lemma 4.5. Assume that $L(z)$ has no multiple roots. Then 


$$
L^{\prime}\left(z_{k}\right)=\frac{\chi_{0}^{2}}{4 \pi^{2} k^{2}\left(a_{k} \bar{b}_{k}\right)}+o\left(\frac{1}{k^{2}\left(a_{k} \bar{b}_{k}\right)}\right) \text { as }|k| \rightarrow \infty
$$

in particular

$$
\left|L^{\prime}\left(z_{k}\right)\right| \geq \frac{C}{k^{2}\left|a_{k} \bar{b}_{k}\right|} \text { for } k= \pm 2, \pm 3, \pm 4, \ldots
$$

and some $C>0$.

Furthermore, there holds

$$
L^{\prime \prime}(\mu)=\frac{2 a_{k} \bar{b}_{k}}{(\mu-2 \pi i k)^{3}}+o\left(\frac{1}{|\mu|^{2}}\right) \text { as } k \rightarrow \infty
$$

uniformly on sets $|\mu-2 \pi i k| \leq \frac{1}{10}$

Proof. It follows from (3.8a) that

$$
\begin{gathered}
L^{\prime}\left(z_{k}\right)=-\frac{a_{k} \bar{b}_{k}}{\left(z_{k}-2 \pi i k\right)^{2}}-\sum_{k \neq \ell} \frac{a_{\ell} \bar{b}_{\ell}}{\left(z_{\ell}-2 \pi i \ell\right)^{2}} \\
\equiv L_{1}+L_{2}
\end{gathered}
$$

We now claim that

$$
\left|\sum_{k \neq \ell} \frac{a_{\ell} \bar{b}_{\ell}}{(z-2 \pi i \ell)^{2}}-\frac{1}{z^{2}} \sum_{-\infty}^{\infty} a_{\ell} \bar{b}_{\ell}\right|=o\left(\frac{1}{|z|^{2}}\right)
$$

as $|k| \rightarrow \infty$ uniformly on sets $|z-2 \pi i k| \leq \frac{1}{10}$

In order to derive (4.25), we observe that

$$
\begin{gathered}
\left|\sum_{k \neq \ell} \frac{a_{\ell} \bar{b}_{\ell}}{(z-2 \pi i \ell)^{2}}-\frac{1}{z^{2}} \sum_{-\infty}^{\infty} a_{\ell} \bar{b}_{\ell}\right| \\
\leq \frac{\left|a_{k} \bar{b}_{k}\right|}{|z|^{2}}+\left|\sum_{k \neq \ell} a_{\ell} \bar{b}_{\ell}\left(\frac{1}{(z-2 \pi i \ell)^{2}}\right)-\frac{1}{z^{2}} \sum_{-\infty}^{\infty} a_{\ell} \bar{b}_{\ell}\right| \\
\leq \frac{\left|a_{k} \bar{b}_{k}\right|}{|z|^{2}}+\frac{1}{|z|^{2}} \sum_{k \neq \ell} \frac{\left(4 \pi|\ell||z|+4 \pi^{2} \ell^{2}\right.}{(z-2 \pi i \ell)^{2}}\left|a_{\ell} \bar{b}_{\ell}\right| \\
\equiv \frac{\left|a_{k} \bar{b}_{k}\right|}{|z|^{2}}+S
\end{gathered}
$$


Therefore, if $|z-2 \pi i k| \leq \frac{1}{10}$ and $|k| \rightarrow \infty$, we can bound the last term as follows

$$
S \leq C \sum_{k \neq \ell}\left(|k \| \ell|+\ell^{2}\right) \frac{\left|a_{\ell} \bar{b}_{\ell}\right|}{|k-\ell|^{2}} \leq C \sum_{k \neq \ell}\left(|k-\ell||\ell|+\ell^{2}\right) \frac{\left|a_{\ell} \bar{b}_{\ell}\right|}{|k-\ell|^{2}}
$$

for some $C>0$ independent of $\mathrm{k}$. Recalling (4.21), we then have that, as $|k| \rightarrow \infty$

$$
\begin{gathered}
L^{\prime}\left(z_{k}\right)=-\frac{a_{k} \bar{b}_{k}}{\left(z_{k}-2 \pi i k\right)^{2}}-\frac{1}{z_{k}} \sum a_{\ell} \bar{b}_{\ell}=o\left(\frac{1}{\left|z_{k}\right|^{2}}\right) \\
=-a_{k} \bar{b}_{k}\left(\frac{-2 \pi i k\left|a_{k} \bar{b}_{k}\right|}{\chi_{0}}+o|k|\left|a_{k} \bar{b}_{k}\right|^{-1}-\frac{\chi_{0}}{(2 \pi i k)^{2}}+o\left(\frac{1}{\left|z_{k}\right|^{2}}\right)\right) \\
=\frac{\chi_{0}^{2}}{4 \pi^{2} k^{2} a_{k} \bar{b}_{k}}(1+o(1))+\frac{\chi_{0}}{4 \pi^{2} k^{2}}+o\left(\frac{1}{|k|^{2}}\right) \\
=\frac{\chi_{0}^{2}}{4 \pi^{2} k^{2} a_{k} \bar{b}_{k}}+o\left(\frac{1}{k^{2} a_{k} \bar{b}_{k}}\right)
\end{gathered}
$$

where use has been made of the fact that $k^{2}\left|a_{k} \bar{b}_{k}\right|=o(1)$ as $|k| \rightarrow \infty$ (since $\left.\sum \ell^{2}\left|a_{\ell} \bar{b}_{\ell}\right|<+\infty\right)$. This yields (4.23a) whence (4.23b) follows. To derive (4.24), we argue in a similar way. We start with

$$
L^{\prime \prime}(z)=\frac{2 a_{k} \bar{b}_{k}}{(z-2 \pi i k)^{3}}+\sum_{k \neq \ell} \frac{2 a_{\ell} \bar{b}_{\ell}}{(z-2 \pi i \ell)^{3}}
$$

Instead of (4.25), we now obtain

$$
\sum_{k \neq \ell} \frac{a_{\ell} \bar{b}_{\ell}}{(z-2 \pi i k)^{3}}=o\left(\frac{1}{k^{2}}\right) \text { as }|k| \rightarrow \infty
$$

uniformly on sets $|z-2 \pi i k| \leq \frac{1}{10}$ which in turn gives at once (4.24). We shall deduce (4.26) after some algebra. Namely, we notice that, as $|k| \rightarrow \infty$,

$$
\begin{gathered}
\left|\sum_{k \neq \ell} \frac{a_{\ell} \bar{b}_{\ell}}{(z-2 \pi i \ell)^{3}}-\frac{1}{z^{3}} \sum_{-\infty}^{\infty} a_{\ell} \bar{b}_{\ell}\right| \\
\leq \frac{\left|a_{k} \bar{b}_{k}\right|}{|z|^{3}}+\sum_{k \neq \ell}\left|a_{\ell} \bar{b}_{\ell}\right|\left|\frac{1}{(z-2 \pi i \ell)^{3}}-\frac{1}{z^{3}}\right| \\
\leq C \sum_{k \neq \ell} \frac{\left(k^{2}|\ell|+\ell^{2}|k|+|\ell|^{3}\right)\left|a_{\ell} \bar{b}_{\ell}\right|}{|k|^{3}|\ell-k|^{3}}+o\left(\frac{1}{|z|^{3}}\right) \\
\equiv S_{1}+o\left(\frac{1}{|z|^{3}}\right)
\end{gathered}
$$


for some generic constant $C>0$. To proceed further, we split $S_{1}$ as follows

$$
S_{1}=S_{1}^{1}+S_{1}^{2}+S_{1}^{3}
$$

where in $S_{1}^{1}$ (resp. in $S_{1}^{2}, S_{1}^{3}$ ) summation is extended to indexes $\ell$ with $|\ell| \geq 2|k|$ (resp. $\left.\frac{|k|}{2} \leq|\ell|<2|k|,|\ell|<\frac{|k|}{2}\right)$. A careful analysis of these terms reveals that

$$
S_{1}=o\left(\frac{1}{k^{2}}\right) \text { as }|k| \rightarrow \infty
$$

Indeed, consider $S_{1}^{2}$, and denote by $\sum_{\ell, k}$ the corresponding summation therein. We then have that

$$
\begin{gathered}
S_{1}^{2}=C \sum_{k, \ell} \frac{\left(k|\ell|+\ell^{2}|k|+|\ell|^{3}\right)\left|a_{\ell} \bar{b}_{\ell}\right|}{|k|^{3}|\ell-k|^{3}} \\
\leq \frac{C}{|k|^{3}} \sum_{k, \ell}|k| \ell^{2}\left|a_{\ell} \bar{b}_{\ell}\right|=o\left(\frac{1}{k^{2}}\right) \text { as }|k| \rightarrow \infty
\end{gathered}
$$

Putting together (4.27) and (4.28), (4.26) follows and the proof is concluded.

We next observe that, by (4.21)

$$
\begin{gathered}
\text { There exists } \theta>0 \text { such that, if } z_{k} \neq 2 \pi i k, \\
\left|z_{k}-2 \pi i k\right| \geq \theta\left|k \| a_{k} \bar{b}_{k}\right| \text { for } k=0, \pm 1, \pm 2, \ldots
\end{gathered}
$$

Suppose now that

$$
\left|\mu-z_{k}\right| \leq \frac{\theta}{2}|k|\left|a_{k} \bar{b}_{k}\right|
$$

Then $|\mu-2 \pi i k| \geq \frac{\theta}{2}|k|\left|a_{k} \bar{b}_{k}\right|$, and (4.24) yields at once.

Corollary 4.6. If (4.30) holds, then

$$
\sup _{\Lambda}\left|L^{\prime \prime}(\mu)\right| \leq \frac{C}{|k|^{3}\left|a_{k} \bar{b}_{k}\right|^{2}}+o\left(\frac{1}{k^{2}}\right) \text { as }|k| \rightarrow \infty
$$

for some $C>0$, where $\Lambda=\left\{\mu \neq 2 \pi i k:\left|\mu-z_{k}\right| \leq \frac{\theta}{2}|k|\left|a_{k} \bar{b}_{k}\right|, \theta\right.$ being as in (3.29) $\}$

Our next step is 
Lemma 4.7. Assume that $L(0) \neq 0$ and $L(z)$ has no multiple roots. Suppose also that $z_{k} \neq 2 \pi i k$, and define

$$
\rho_{k}=\frac{2}{\left|L^{\prime}\left(z_{k}\right)\right|} \frac{\left|z_{k}-z\right|}{\left|Q z_{k} z\right|}\left(\left|1+\frac{z_{k}}{z}\right|+A\right)
$$

where $A$ is a fixed positive constant. Then there exists $C>0$ (independent of Re, $k$ ) such that

$$
\begin{gathered}
\left|L(\mu)-L^{\prime}\left(z_{k}\right)\left(\mu-z_{k}\right)\right| \leq \frac{C|k|^{3}}{|Q|^{2}} \text { for } k= \pm 1, \pm 2, \ldots \\
\text { provided that }\left|\mu-z_{k}\right|=\rho_{k} \text { and }|k| \leq C R e
\end{gathered}
$$

Proof. Since $L(0) \neq 0$, one has that $z_{k} \neq 0$ for any $k$. By (4.23b)

$$
\rho_{k} \leq C K^{2}\left|a_{k} \bar{b}_{k}\right| \frac{\left|z_{k}-z\right|}{\left|Q z_{k} z\right|}\left(\left|1+\frac{z_{k}}{z}\right|+A\right) \equiv B_{k}
$$

where here and henceforth $C$ will denote a generic constant which is independent of $R e$ and $\mathrm{k}$. We want to have

$$
B_{k} \leq \frac{\theta}{2}|k|\left|a_{k} \bar{b}_{k}\right|
$$

where $\theta$ is the constant in (4.29). This last inequality holds provided that

$$
\frac{2 C}{\theta} \cdot \frac{\left|z_{k}-z\right||k|}{|Q|\left|z_{k} z\right|}\left(\left|1+\frac{z_{k}}{z}\right|+A\right) \leq 1
$$

Taking into account (4.16) as well as the fact that $z=O(1)$ for $R e>>1,(4.34 \mathrm{~b})$ will hold if $|k| \leq \sigma R e$ for some $\sigma>0$ and $R e$ is large enough. Then, if $\left|\mu-z_{k}\right| \leq \rho_{k}$ and $\Lambda$ is the set defined in Corollary 4.6, we will have that, as $|j| \rightarrow \infty$

$$
\begin{gathered}
\left|L(\mu)-L^{\prime}\left(z_{k}\right)\left(\mu-z_{k}\right)\right| \leq \frac{1}{2}\left|\sup _{\Lambda} L^{\prime \prime}(\mu)\right|\left|\mu-z_{k}\right|^{2} \\
\leq \frac{\rho_{k}^{2}}{2}\left(\frac{C}{|k|^{3}\left|a_{k} \bar{b}_{k}\right|^{2}}+o\left(\frac{1}{k^{2}}\right)\right) \\
\leq \frac{1}{2}\left(\frac{C^{2} k^{4}\left|a_{k} \bar{b}_{k}\right|^{2}\left|z_{k}-z\right|^{2}}{\left|Q z_{k} z\right|^{2}}\left|1+\frac{z_{k}}{z}\right|^{2}\right)\left(\frac{C}{|k|^{3}\left|a_{k} \bar{b}_{k}\right|^{2}}+o\left(\frac{1}{k^{2}}\right)\right) \\
\leq \frac{C|k|^{3}}{|Q|^{2}|z|^{2}}+o\left(\frac{k^{4}\left|a_{k} \bar{b}_{k}\right|^{2}}{|Q|^{2}|z|^{2}}\right),
\end{gathered}
$$


so that (4.33) follows.

\subsection{The proof of Theorem 4.3.}

Lemmata 4.4-4.7 have proved the ground to tackle the proof of our result on the stability of slow solutions. To begin with, we are now in a position to show a particular case of (4.18), (4.19), namely

Lemma 4.8. Assume that the hypotheses in Theorem 3.2 hold. Suppose also that (3.16) and (4.17) are satisfied. Then the roots $\left\{\mu_{k}\right\}$ of $\left(1-e^{\mu}\right) S(\mu)$ with $k= \pm 1, \pm 2, \pm 3, . . k \neq j$, are such that

$$
\left|\mu_{k}-z_{k}\right|=o(1) \text { for }|k| \leq(R e)^{1 / 3} \text { as } R e \rightarrow \infty
$$

On the other hand, there is one root $\mu_{0}$ such that

$$
\left|\mu_{0}\right|=o(1), \text { as } R e \rightarrow \infty
$$

Proof. It clearly suffices to consider the case where $a_{k} \bar{b}_{k} \neq 0$. Assume first that $L(0) \neq 0$, and let $\rho_{k}$ be as in (4.32). Then (4.35a) will follow as soon as we prove that

$$
\begin{gathered}
\text { If }\left|\mu-z_{k}\right|=\rho_{k}, \text { then } \\
\left|S(\mu)-\left(\frac{\mathrm{Qzz}_{k}}{z_{k}-z}\right) L^{\prime}\left(z_{k}\right)\left(\mu-z_{k}\right)\right|<2\left(\left|1+\frac{z_{k}}{z}\right|+A\right)
\end{gathered}
$$

To derive (4.36), we just observe that, if $\left|\mu-z_{k}\right|=\rho_{k}$,

$$
\begin{aligned}
& \left|S(\mu)-\left(\frac{\mathrm{Qzz}_{k}}{z_{k}-z}\right) L^{\prime}\left(z_{k}\right)\left(\mu-z_{k}\right)\right| \\
& \leq 1+\frac{|\mu|}{|z|}+\epsilon\left|Q v^{2}\right|\left|\frac{\mu z}{\mu-z}\right|+C \frac{|Q \mu z|}{|\mu-z|} \frac{|k|^{3}}{|Q|^{2}} \\
& +\left|L^{\prime}\left(z_{k}\right)\left\|\mu-z_{k}\right\| Q \| z\right|\left|\frac{\mu}{\mu-z}-\frac{z_{k}}{z_{k}-z}\right| \\
& \equiv I_{1}+I_{2}+I_{3}+I_{4}
\end{aligned}
$$

for some $C>0$. Taking into account that $|v| \approx R e$ as $R e \rightarrow \infty$ we readily see that 


$$
\sum_{i=1}^{4} I_{i} \leq 1+\frac{|\mu|}{|z|}+B+\frac{C|k|^{3}}{|Q|}+\frac{\left|L^{\prime}\left(z_{k}\right)\right||Q||z|^{2}\left|\mu-z_{k}\right|^{2}}{|\mu-z|\left|z-z_{k}\right|}
$$

We now relate $k$ and $R e$ as follows

$$
|k| \leq(R e)^{1 / 3}
$$

Notice that this relation is compatible with the one in (4.33). Since $\rho_{k} \rightarrow 0$ as $R e \rightarrow \infty$, one then has

$$
\sum_{i=1}^{4} I_{i} \leq M+1+\frac{|\mu|}{|z|} \text { if } R e>>1
$$

for some $M>0$ large enough. Taking then $A=2 M,(4.36)$ follows. As a matter of fact, by our previous estimates we have shown that

$$
\begin{gathered}
\rho_{k} \leq C k^{2}\left|a_{k} \bar{b}_{k}\right| \frac{\left|z_{k}-z\right|}{|Q||z|\left|z_{k}\right|}\left(\left|1+\frac{z_{k}}{z}\right|+A\right) \\
\leq C_{1} \frac{\left(1+|k|^{3}\right)\left|a_{k} \bar{b}_{k}\right|}{|Q|} \leq C_{1} \frac{\left(1+k^{2}\right)\left|a_{k} \bar{b}_{k}\right| R e^{1 / 3}}{|Q|} \\
\leq C_{1}\left(1+k^{2}\right)\left|a_{k} \bar{b}_{k}\right|(R e)^{-5 / 3}
\end{gathered}
$$

for some $C_{1}$ depending on $A$ and $z$. Hence

$$
\left|\mu_{k}-z_{k}\right| \leq C\left(1+k^{2}\right)\left|a_{k} \bar{b}_{k}\right|(R e)^{-5 / 3} \text {, whenever }
$$

$|k| \leq(R e)^{1 / 3}, k= \pm 1, \pm 2, \pm 3, \ldots$ and $R e$ is large enough.

To derive (4.35b) when $L(0) \neq 0$, we modify our previous argument as follows. We consider the curves $\Gamma=\left\{\mu:|\mu|=\frac{3}{|Q||L(0)|}\right\}$, and obtain after standard computations that

$$
|S(\mu)-(1-Q L(0) \mu)|<|1-Q L(0) \mu| \text { on } \gamma
$$

whereupon we conclude as before. Finally, if $L(0)=0$, there is a root $z_{0}=0$ of $L(z)$ which cannot be dealt with as before. For those roots approaching $z_{k}$ with $k \neq 0$, the previous argument still yields the result. The roots $\mu=o(1)$ are kept track of just by replacing $(1-Q L(0) \mu)$ by $\left(1-Q L^{\prime}(0) \mu^{2}\right)$ above.

We now proceed to remove the assumption $|k| \leq(R e)^{1 / 3}$ in $(4.35 \mathrm{a})$. To this end, we prove 
Lemma 4.9. Let $D_{k}=\left\{\mu \in \mathbb{C}: 2 \pi\left(|k|-\frac{1}{2}\right)<|\mu|<2 \pi\left(|k|+\frac{1}{2}\right)\right\}$. Then there holds

$$
\begin{gathered}
\sup _{\mu \in D_{k}} \mu^{2}\left|L(\mu)-\frac{a_{k} \bar{b}_{k}}{\mu-2 \pi i k}-\frac{\chi_{0}}{\mu}-\frac{M_{0}}{\mu^{2}}\right| \rightarrow 0 \\
\text { as }|k| \rightarrow \infty .
\end{gathered}
$$

where $M_{0}$ and $\chi_{0}$ are as in the statement of Theorem 4.3.

Proof. We start from

$$
L(\mu)=\frac{a_{k} \bar{b}_{k}}{\mu-2 \pi i k}+\sum_{k \neq \ell} \frac{a_{\ell} \bar{b}_{\ell}}{\mu-2 \pi i \ell}
$$

and compute

$$
\begin{gathered}
\sum_{k \neq \ell} \frac{a_{\ell} \bar{b}_{\ell}}{\mu-2 \pi i \ell}=\frac{\chi_{0}}{\mu}+\frac{1}{\mu} \sum_{k \neq \ell} \frac{2 \pi i \ell a_{k} \bar{b}_{k}}{\mu-2 \pi i \ell}-\frac{a_{k} \bar{b}_{k}}{\mu} \\
\left|\sum_{k \neq \ell} \frac{2 \pi i \ell a_{k} \bar{b}_{k}}{\mu-2 \pi i \ell}-\frac{M_{0}}{\mu}\right| \leq \frac{4 \pi|k|\left|a_{k} \bar{b}_{k}\right|}{|k|}+\frac{4 \pi^{2}}{|k|} \sum_{k \neq \ell} \frac{\ell^{2}\left|a_{\ell} \bar{b}_{\ell}\right|}{|\mu-2 \pi i \ell|}
\end{gathered}
$$

since $|\mu-2 \pi i \ell| \geq \theta>0$ for some $\theta$ whenever $\mu \in\left\{\mathbb{C}: 2 \pi\left(|k|-\frac{1}{2}\right)<|\mu|<2 \pi\left(|k|+\frac{1}{2}\right)\right\}$ we also have that

$$
\lim _{|k| \rightarrow \infty}\left(\sum_{k \neq \ell} \frac{\ell^{2}\left|a_{\ell} \bar{b}_{\ell}\right|}{|\mu-2 \pi i \ell|}\right)=0 .
$$

Putting all these estimates together (4.39) follows.

We are now prepared to show

Lemma 4.10. Under the assumptions of Lemma 4.8, there holds

$$
\left|\mu_{k}-z_{k}\right|=o(1) \text { as } R e \rightarrow \infty \text { for } k=0, \pm 1, \pm 2, \ldots
$$

Proof. It involves a number of tedious (but otherwise routine) computations. We shall therefore sketch the main lines in the argument and leave most of the details to the reader. Clearly, it suffices to examine the case where $|k| \geq(R e)^{1 / 3}$. Consider the function 


$$
G_{k}(\mu)=\frac{\mu}{z}-\frac{Q z \chi_{0}}{\mu}+Q z\left(\epsilon v^{2}-\frac{M_{0}}{\mu^{2}}\right)-\frac{\mathrm{Qza}_{k} \bar{b}_{k}}{\mu-2 \pi i k}
$$

Using (4.39), we then have that

$$
\begin{aligned}
&\left|S(\mu)-G_{k}(\mu)\right| \leq \frac{C}{|\mu|}\left(1+\frac{|Q|\left|a_{k} \bar{b}_{k}\right|}{|\mu-2 \pi i k|}\right)+\left|1+\frac{Q z^{2} \chi_{0}}{|\mu|^{2}}\right| \\
&+o\left(\frac{|Q|}{|\mu|^{2}}\right) \text { as }|j| \rightarrow \infty, \\
& \text { uniformly for }|\mu-2 \pi i k| \leq \frac{1}{10}
\end{aligned}
$$

Set now

$$
\begin{gathered}
\rho_{k}=2\left|Q a_{k} \bar{b}_{k}\right|\left(\left|Q z\left(\epsilon v^{2}+\frac{M_{0}}{4 \pi^{2} k^{2}}\right)+i\left(\frac{2 \pi k}{z}+\frac{Q z \chi_{0}}{2 \pi k}\right)\right|\right)^{-1} \\
\equiv 2\left|\operatorname{Qza}_{k} \bar{b}_{k}\right| H^{-1}
\end{gathered}
$$

Notice that

$$
\begin{gathered}
\text { If }\left|\frac{2 \pi k}{z}+\frac{Q z \chi_{0}}{2 \pi k}\right| \geq \frac{1}{2}, \text { then }|H| \geq \frac{1}{2}, \\
\text { If }\left|\frac{2 \pi k}{z}+\frac{Q z \chi_{0}}{2 \pi k}\right| \leq \frac{1}{2}, \text { then } C_{1} k^{2} \leq|Q| \leq C_{2} k^{2}
\end{gathered}
$$

for some constants $C_{1}, C_{2}$ independent of $R e$ and $k$

On the other hand, we readily see that

$$
\begin{gathered}
|H| \geq\left|Q z\left(\epsilon v^{2}+\frac{M_{0}}{4 \pi^{2} k^{2}}\right)\right| \\
\geq \mid Q z\left(\epsilon v^{2}+M_{0}\left(Q z^{2} \chi_{0}+4 \pi^{2} k^{2}-Q z^{2} \chi_{0}\right)^{-1} \mid\right. \\
\geq\left|Q z \epsilon v^{2}+M_{0}\left(Q z^{2} \chi_{0}\right)^{-1}\right| \\
\left.-\mid Q_{z M_{0}}\left(Q z^{2} \chi_{0}+4 \pi^{2} k^{2}-Q z^{2} \chi_{0}\right)^{-1}-\left(Q z^{2} \chi_{0}\right)^{-1}\right) \mid \\
\geq\left|Q \epsilon v^{2} z+\frac{M_{0}}{\chi_{0} z}\right|-C \frac{M_{0}|k|}{|Q| \chi_{0}^{2}}
\end{gathered}
$$


for some $C>0$. Since the second term above behaves asymptotically as $|k|^{-1}$ for large $|k|$, we obtain that

$$
|H| \geq C_{1}>0 \text { as } R e \rightarrow \infty, \text { where } C_{1} \text { is }
$$

independent of $\mathrm{k}$.

We next claim that

$$
\begin{gathered}
\rho_{k} \leq C\left|k \| a_{k} \bar{b}_{k}\right| \text { for some } C>0, \text { so that } \\
\rho_{k} \rightarrow 0 \text { if }|k| \geq R e^{1 / 3} \text { and } R e \rightarrow \infty .
\end{gathered}
$$

Estimate (4.46) is obtained by arguments alike to those in (4.44). Our next goal is to show that

$$
\begin{gathered}
\left|G_{k}(\mu)\right|>>\left|S(\mu)-G_{k}(\mu)\right| \text { as } R e \rightarrow \infty, \text { uniformly for } \\
|\mu-2 \pi i k|=\rho_{k} \text { with }|k| \geq R e^{1 / 3}
\end{gathered}
$$

To derive (4.47), we set $\xi=\mu-2 \pi i k$, so that $\frac{Q z \chi_{0}}{\mu}=\frac{Q z \chi_{0}}{2 \pi i k}\left(1-\frac{\xi}{2 \pi i k}+\ldots\right)$ in the region under consideration. We have there that

$$
\begin{gathered}
\left|G_{k}(\mu)\right|=\mid \frac{2 \pi i k}{z}-\frac{Q z \chi_{0}}{2 \pi i k}+\frac{\xi}{z}+O\left(\frac{\left|Q z \chi_{0}\right||\xi|}{k^{2}}\right) \\
+\frac{\mathrm{Q}^{2} \mathrm{M}_{0}}{4 \pi^{2} k^{2}}+Q \epsilon v^{2} z+O\left(\frac{\left|\mathrm{QzM}_{0}\right||\xi|}{|k|^{3}}\right)-\frac{\mathrm{Qza}_{k} \bar{b}_{k}}{\xi} \mid \\
\geq\left|Q z\left(\epsilon v^{2} \frac{M_{0}}{4 \pi^{2} k^{2}}\right)+i\left(\frac{2 \pi k}{z}+\frac{Q z \chi_{0}}{2 \pi k}\right)\right|-\frac{\mathrm{Qza}_{k} \bar{b}_{k}}{|\xi|} \\
-|\xi|\left(\frac{1}{|z|}+O\left(\frac{\left|Q z \chi_{0}\right|}{k^{2}}\right)\right) \\
\geq \frac{1}{4}\left|Q z\left(\epsilon v^{2}+\frac{M_{0}}{4 \pi^{2} k^{2}}\right)+i\left(\frac{2 \pi k}{z}+\frac{Q z \chi_{0}}{2 \pi k}\right)\right| \\
>>\left|S(\mu)-G_{k}(\mu)\right|
\end{gathered}
$$

where we have used (4.42) to derive the last statement above. By the argument principle, we then deduce that 
As $R e \rightarrow \infty$, the difference between the number of zeroes and poles in the region $|\mu-2 \pi i k| \leq \rho_{k}\left(|k| \leq R e^{1 / 3}\right)$, is the same for functions $S(\mu)$ and $G_{k}(\mu)$.

As a further step, we now consider function $\tilde{G}_{k}(\mu)$ given by

$$
\tilde{G}_{k}(\mu)=\frac{2 \pi k}{z}-\frac{Q z \chi_{0}}{2 \pi i k}+Q z\left(\epsilon v^{2}+\frac{M_{0}}{4 \pi^{2} k^{2}}\right)-\frac{\mathrm{Qza}_{k} \bar{b}_{k}}{\mu 2 \pi i k}
$$

It is readily seen that

$$
\begin{aligned}
\left|\tilde{G}_{k}(\mu)\right| \geq \mid Q z\left(\epsilon v^{2}\right. & \left.+\frac{M_{0}}{4 \pi^{2} k^{2}}\right)+i\left(\frac{2 \pi k}{z}+\frac{Q z \chi_{0}}{2 \pi k}\right) \mid- \\
& -\frac{\left|\mathrm{Qza}_{k} \bar{b}_{k}\right|}{\left|\rho_{k}\right|}
\end{aligned}
$$

whereas, if $\xi=\mu-2 \pi i k$,

$$
\left|G_{k}(\mu)-\tilde{G}_{k}(\mu)\right| \leq C\left(\frac{1}{|z|}+|Q| k^{2}+\frac{|Q| M_{0}}{|k|^{3}}\right)|\xi|
$$

for some $C>0$. We then deduce that, if $|\mu-2 \pi i k|$ with $R e>>1,\left|G_{k}(\mu)-\tilde{G}_{k}(\mu)\right|<<$ $\left|G_{k}(\mu)\right|$ whence

As $R e \rightarrow \infty$, the difference between the number of zeroes and poles in the region $|\mu-2 \pi i k| \leq \rho_{k}\left(|k| \geq R e^{1 / 3}\right)$ is the same for functions $G_{k}(\mu)$ and $\tilde{G}_{k}(\mu)$.

From (4.48) and (4.49), we deduce that $S(\mu)$ has one zero $\mu_{k}$ such that $\left|\mu_{k}-2 \pi i k\right| \leq \rho_{k}$. From this and (3.9), (4.40) follows.

The last result in this Section is

Lemma 4.11. There exists two roots $\mu_{ \pm 1}$ of $S(\mu)$ such that

$$
\begin{gathered}
\mu_{ \pm 1}= \pm\left(Q z^{2} \chi_{0}\right)^{1 / 2}-\frac{\epsilon z^{2} q}{2}\left(\xi_{j}^{\ell}\right)^{2}+\frac{M_{0}}{2 \chi_{0}} \\
+o(1) \text { as } R e \rightarrow \infty
\end{gathered}
$$

where 


$$
q=\lim _{R e \rightarrow \infty} \frac{Q}{R e^{2}}
$$

Proof. Let us define

$$
G= \pm\left(Q z^{2} \chi_{0}\right)^{1 / 2}
$$

Clearly, $G \approx$ CRe as $R e \rightarrow \infty$ for some $C>0$. Consider now the region

$$
\sum=\left\{\mu:|\mu-G|=\left|\frac{z}{2}\left(\frac{M_{0}}{\chi_{0} z}+\epsilon \mathrm{Qzv}^{2}\right)+\rho e^{i \theta}\right|\right\}
$$

where $\rho>0$ will be selected presently. Once again, we shall make use of Rouche's Theorem to derive the result. To this end, we consider the auxiliary function

$$
F(\mu)=\frac{2}{z}(\mu-G)-\left(\frac{M_{0}}{\chi_{0} z}-\epsilon \mathrm{Qzv}^{2}\right)
$$

Arguing as in Lemma 4.9, we readily see that

$$
\begin{gathered}
L(\mu)=\frac{\chi_{0}}{\mu}+\frac{M_{0}}{\mu^{2}}+g(\mu), \text { where } g(\mu)=o\left(|\mu|^{-2}\right) \\
\text { as }|\mu| \rightarrow \infty .
\end{gathered}
$$

We now claim that

$$
|S(\mu)-F(\mu)|<|F(\mu)| \text { in } \sum
$$

whereupon (4.50) follows. The above inequality is obtained much in the same way as many of our previous estimates. We shall therefore omit most of the details involved. We merely point out that in view of (4.51), we have that

$$
\begin{gathered}
S(\mu)-F(\mu)=-Q z\left(\frac{\chi_{0}}{\mu}+\frac{M_{0}}{\mu^{2}}+g(\mu)\right)-\frac{Q z^{2} \mu_{0}}{\mu^{2}} \\
+O\left(\frac{1}{|\mu|}\right)+1-\frac{\mu}{z}+\frac{2 G}{z}+\frac{M_{0}}{\chi_{0} z}
\end{gathered}
$$

as $R e \rightarrow \infty$, whence

$$
|S(\mu)-F(\mu)| \leq|Q\|z\| g(\mu)|+O\left(\frac{1}{|\mu|}\right) \text { on } \sum
$$


as $R e \rightarrow \infty$, whereas on the other hand

$$
|F(\mu)|=\frac{2}{|z|} \rho \text { on } \sum
$$

so that selecting now $\rho=C_{1} \max \left\{|\mu|^{2} g(\mu),|\mu|^{-1 / 2}\right\}$ for some suitable $C_{1}>0,(4.52)$ follow.

Putting together Lemmata 4.8-4.11, the proof of Theorem 4.3 is now complete.

\subsection{On the existence of linearly stable slow solutions}

We have already shown that fast solutions (if any) are always linearly stable (cf. Corollary 4.2). On the other hand, Theorem 4.3 provides a description of the asymptotics of the eigenvalues corresponding to linearizing around a given stationary solution. It is remarkable that, for most of such eigenvalues, their asymptotic behaviour is encoded in the zeroes of the function $L(z)$ (cf. (4.18)). A question which naturally arises is whether functions $f$ and $T_{w}$ can be selected so that linearly stable solutions satisfying (3.16) exist. An affirmative answer is provided by the following result

THEOREM 4.12. There exists a set of functions $f$ and $T_{w}$ for which there are some linearly stable slow solutions and two stable fast solutions.

For convenience, we shall deduce Theorem 4.12 from two technical results. Set $\Gamma_{k}=a_{k} \bar{b}_{k}$, where $a_{k}$ and $b_{k}$ are given in (3.4). We then have

Lemma 4.13. Let $L(z)$ be the function given in (3.8) and let $\tilde{z}_{j} ; j=0, \pm 2, \pm 3, \pm 4, \ldots$ the sequence of its zeroes. Fix $k=0, \pm 2, \pm 3, \pm 4, \ldots$ and a set of autoconjugate numbers $\tilde{z}_{j}$ $j=0, \pm 2, \pm 3, \pm 4, \ldots \pm k$. Then there exists a function $\tilde{L}(z)$ such that $\tilde{L}(z)=\sum_{\infty}^{\infty} \frac{\tilde{\Gamma}_{k}}{z-2 \pi k i}$, where $\bar{\Gamma}_{k}=\tilde{\Gamma}_{-k}, \sum_{-\infty}^{\infty} k^{2}\left|\tilde{\Gamma}_{k}\right|<+\infty$, and the set of zeroes of $\tilde{L}(z)$ is given by

$$
\tilde{S}=\left\{\tilde{z}_{j} ; j=0, \pm 2, \ldots, \pm k\right\} \bigcup\left\{z_{j}: j= \pm k+1, \ldots\right\}
$$

Proof. Set

$$
\tilde{L}(z)=\prod_{j=-k}^{j=+k}\left(\frac{z-\tilde{z}_{j}}{z-z_{j}}\right) L(z)=\sum_{\ell=-\infty}^{\ell=+\infty} \prod_{j=-k}^{j=+k}\left(\frac{z-\tilde{z}_{j}}{z-z_{j}}\right) \frac{\Gamma_{\ell}}{z-2 \pi i \ell}
$$

Clearly, $\tilde{L}(z)$ is a meromorphic function having poles at most at points $z=2 \pi i \ell$. Define now 


$$
\tilde{\Gamma}_{\ell}=\lim _{z-2 \pi i \ell}(\tilde{L}(z)(z-2 \pi i \ell))=\prod_{j=-k}^{j=+k}\left(\frac{2 \pi i j-\tilde{z}_{j}}{2 \pi i j-z_{j}}\right) \Gamma_{\ell}
$$

It then follows that $R(z)=\tilde{L}(z)-\sum_{-\infty}^{\infty} \frac{\tilde{\Gamma}_{\ell}}{z-2 \pi i \ell}$ is an entire function, and since $R(z)=o(1)$ when $|z|=(2 N+1) \pi$ and $N \rightarrow \infty$, we then deduce that $R(z) \equiv 0$.

We deduce from Lemma 4.13 that, for any given pair of functions $f$ and $T_{w}$, we can modify their Fourier coefficients (thus changing the geometry of the pipe and the temperature at the wall) so that

i) For the new data $\tilde{f}$ and $\tilde{T}_{w}$, the corresponding function $\tilde{L}(z)$ has exactly $2 J+1$ zeroes $\left\{z_{-J}, z_{-J+1}, \ldots, z_{0}, \ldots, z_{J-1}, z_{J}\right\}$ lying on the real axis.

ii) When linearization is performed around velocity values satisfying (3.16) with $j=J$, the eigenvalues corresponding to (4.18) and (4.19) are positive.

iii) $L\left( \pm \frac{1}{\epsilon}\right)>0$ and $|z| \pm J \in\left[\frac{m}{\epsilon}, \frac{M}{\epsilon}\right]$.

To discuss the situation corresponding to eigenvalues satisfying (4.20), we need yet to evaluate how parameters $\chi_{0}$ and $M_{0}$ change under transformations of type (4.53). The information required is contained in the following result.

LEMмA 4.14. Let $N$ be a positive integer, $\alpha>1, \nu>0$, and let $\left\{r_{j}\right\},-\infty<j<+\infty$, be a sequence of real numbers such that $r_{j}=-r_{-j}$ for any $j$. For any integer $k$, we set

$$
\tilde{\Gamma}_{k}^{N}=\left(\prod_{N \leq j \leq \alpha N}\left(\frac{2 \pi i(k-j)-\epsilon_{j}}{2 \pi i k-z_{j}}\right)\right) \Gamma_{k} \equiv R_{N, k} \cdot \Gamma_{k}
$$

where $\Gamma_{k}$ as in our previous Lemma, and

$$
\epsilon_{j}=\frac{\nu}{|j|}+r_{j}
$$

Assume now that $\chi_{0} \neq 0$, and define $\tilde{\chi}_{0}^{N}, \tilde{M}_{0}^{N}$ as follows

$$
\tilde{\chi}_{0}^{N}=\sum_{\infty}^{\infty} \tilde{\Gamma}_{k}^{N}, \tilde{M}_{0}^{N}=\sum_{\infty}^{\infty} 2 \pi i k \tilde{\Gamma}_{k}
$$

We then have that

$$
\lim _{n \rightarrow \infty} \tilde{\chi}_{0}^{N}=\chi_{0}
$$




$$
\lim _{n \rightarrow \infty} \tilde{M}_{0}^{N}=M_{0}-\chi_{0} \nu \log \alpha
$$

Proof. We shall prove first (4.55). To this end, we set $\delta_{j}=z_{j}-2 \pi i j$. By (4.25), we then have that

$$
\delta_{j}=-\frac{2 \pi i j}{\chi_{0}} \Gamma_{j}+o\left(\left|j \| \Gamma_{j}\right|\right) \text { as }|j| \rightarrow \infty
$$

We now split $\tilde{\chi}_{0}^{N}$ as follows

$$
\begin{gathered}
\tilde{\chi}_{0}^{N}=\sum_{\infty}^{\infty} R_{N, k} \Gamma_{k}=\sum_{k \mid<N} R_{N, k} \Gamma_{k}+\sum_{N \leq|k| \leq \alpha N} R_{N, K} \Gamma_{k} \\
+\sum_{\alpha N<|k|} R_{N, k} \Gamma_{k} \equiv J_{1}^{N}+J_{2}^{N}+J_{3}^{N}
\end{gathered}
$$

Assume now that $|k|<\frac{N}{2}$ or $|k|>2 \alpha N$. Then $|k-j| \geq \frac{N}{2}$ and $|k-j| \geq C|j|$, where here and henceforth $C$ will denote a generic constant (changing possibly from line to line), which is independent of $k$ and $j$. We then have that

$$
\begin{array}{r}
\log R_{N, k}=\sum_{N \leq|j| \leq \alpha N} \log \left(1+\frac{\delta_{j}-\epsilon_{j}}{2 \pi i(k-j)-\delta_{j}}\right) \\
\leq C\left(\sum_{N \leq|j| \leq \alpha N}\left|\delta_{j}\right|+\sum_{N \leq|j| \leq \alpha N} \frac{\left|\epsilon_{j}\right|}{|j|}\right)
\end{array}
$$

Recalling the well known formula

$$
\sum_{j=1}^{n} \frac{1}{j}=\log n+\gamma+o(1) \text { as } n \rightarrow \infty
$$

where $\gamma$ is Euler's constant, we obtain

$$
\log R_{N, k} \leq 2 \log \alpha+o(1) \text { as } N \rightarrow \infty
$$

uniformly when $|k|<\frac{N}{2}$ or $|k|>2 \alpha N$.

Suppose now that $\frac{N}{2} \leq|k|<\mathrm{N}$. Arguing as before, we obtain 


$$
\log R_{N, k} \leq C \delta(\log (\alpha N-N+1)-\gamma)+o(1)
$$

as $N \rightarrow \infty$, where $\delta>0$ can be taken so small that,

$$
\text { say, } C \delta<\frac{1}{5}
$$

A similar argument applies to the case $N \leq|k| \leq 2 \alpha \mathrm{N}$. We thus arrive at

$$
\begin{gathered}
\left|R_{N, k}\right| \leq C|k|^{1 / 5} \text { as } N \rightarrow \infty, \text { uniformly } \\
\text { when } \frac{N}{2} \leq|k| \leq 2 \alpha N
\end{gathered}
$$

Define now $\mu_{N, k}=R_{N, k}$ if $|k|<N$ or $|k|>\alpha N, \mu_{N, k}=0$ otherwise. Clearly,

$$
J_{1}^{N}+J_{3}^{N}=\sum_{-\infty}^{\infty} \mu_{N, k} \Gamma_{k}
$$

Taking into account (4.58) and (4.59), it follows from Lebesgue's Theorem that

$$
\lim _{N \rightarrow \infty}\left(J_{1}^{N}+J_{3}^{N}\right)=\sum_{-\infty}^{\infty}\left(\lim _{n \rightarrow \infty} \mu_{n, k}\right) \Gamma_{k}=\sum_{-\infty}^{\infty}\left(\lim _{n \rightarrow \infty} R_{n, k}\right) \Gamma_{k}
$$

where limits within the summation terms above are to be considered for fixed $\mathrm{k}$. We now claim that

$$
\text { For any fixed } \mathrm{k}, \lim _{n \rightarrow \infty} R_{n, k}=1
$$

To check (4.59), we notice that if we set $\sum_{1}=\sum_{N \leq|j| \leq \alpha N}$, for fixed $k$ and large enough $N$ there holds

$$
\begin{aligned}
\log R_{N, k} & =\sum_{1}\left(\frac{\delta_{j}-\epsilon_{j}}{2 \pi i(k-j)-\delta_{j}}+O\left(\frac{\left(\delta_{j}-\epsilon_{j}\right)^{2}}{(k-j)^{2}}\right)\right) \\
= & \sum_{1} O\left(\frac{\left|\delta_{j}\right|+\left|\epsilon_{j}\right|}{|j|}+\left(\delta_{j}^{2}+\left|\epsilon_{j}\right|\left|\delta_{j}\right|+\frac{\epsilon_{j}^{2}}{j^{2}}\right)\right) \\
& =o(1) \text { as } n \rightarrow \infty
\end{aligned}
$$

whence (4.60). We have therefore proved that 


$$
\lim _{n \rightarrow \infty} J_{1}^{N}+J_{3}^{N}=\sum_{-\infty}^{\infty} \Gamma_{k}=\chi_{0}
$$

The next step consists in showing that

$$
\lim _{n \rightarrow \infty} J_{2}^{N}=0 .
$$

As soon as this had been done, (4.55) will follow from (4.57), (4.61) and (4.62). To prove (4.62), let us write

$$
R_{N, k}=\frac{\epsilon_{k}}{\delta_{k}}\left(\prod_{2} \frac{2 \pi i(k-j)-\epsilon_{j}}{2 \pi i(k-j) \bar{j} \delta_{j}}\right) \equiv \frac{\epsilon_{k}}{\delta_{k}} S_{N, k}
$$

where the symbol $\prod_{2}$ denotes that the product there is extended to indexes $j$ such that $j \neq k, N \leq|j| \leq \alpha N$. Clearly, $\bar{R}_{N, k}=R_{N, k}$ hence

$$
J_{2}^{N}=\sum_{1} R_{N, k} \Gamma_{k}+\left(\overline{\sum_{1} R_{N, k} \Gamma_{k}}\right) \equiv L_{N}+\bar{L}_{N}
$$

Let us define $\sum_{2}$ in a similar way to $\sum_{1}$ above. Then, for fixed $k>0$ and large $N$,

$$
\begin{aligned}
\log \left(S_{N, k}\right) & =\sum_{2} \log \left(1+\frac{\delta_{j}-\epsilon_{j}}{2 \pi i(k-j)-\delta_{j}}\right) \\
& =\sum_{2} \log \left(1+\frac{\epsilon_{j}}{2 \pi i(k-j)}\right)+o(1)
\end{aligned}
$$

Let us denote by $[\sigma]$ the entire part of the real positive number $\sigma$, and assume that $k \neq N, k \neq[\alpha N]$. We now split $\sum_{2}$ in the form $\sum_{2}=\sum_{2,1}+\sum_{2,2}+\sum_{2,3}$, where summation in $\sum_{2,1}$ (resp. $\sum_{2,2}, \sum_{2,3}$ ) is extended to the indexes $N \leq|j| \leq k-1$ (resp. $k+1 \leq j \leq[\alpha N],-[\alpha N] \leq j<N)$. This yields

$$
\begin{aligned}
\log \left(S_{N, k}\right) & =\sum_{\ell=1}^{k-N} \log \left(1-\frac{\epsilon_{k-\ell}}{2 \pi i \ell}\right) \sum_{\ell=1}^{[\alpha N]-k} \log \left(1+\frac{\epsilon_{\ell+k}}{2 \pi i \ell}\right) \\
& +\sum_{\ell=1}^{[\alpha N]-k} \log \left(1+\frac{\epsilon_{\ell-k}}{2 \pi i \ell}\right)+o(1) \\
& \equiv K_{1}^{N}+K_{2}^{N}+K_{3}^{N}+o(1)
\end{aligned}
$$


It is readily seen that, as $N \rightarrow \infty$

$$
\begin{aligned}
K_{3}^{N} & =\sum_{\ell=N+k}^{[\alpha N]+k}\left(-\frac{\epsilon_{k-\ell}}{2 \pi i \ell}+O\left(\left(\frac{\epsilon_{k-\ell}}{\ell}\right)^{2}\right)\right) \\
& =o(1)\left(\sum_{\ell=N+k}^{[\alpha N]+k}\left(\frac{1}{\ell}+\frac{1}{\ell^{2}}\right)\right)=o(1) 1+\log \left(\frac{[\alpha N]+k}{N+k}\right)
\end{aligned}
$$

In a similar way, we obtain that, as $N \rightarrow \infty$

$$
K_{1}^{N}+K_{2}^{N}=\sum_{1}^{k-N}\left(-\frac{\epsilon_{k-\ell}}{2 \pi i \ell}\right)+\sum_{1}^{[\alpha N]-k} \frac{\epsilon_{k+\ell}}{2 \pi i \ell}+o(1)
$$

If we now suppose that $k-1 \neq N, k+1 \neq[\sigma N]$, and set $\beta_{N, k}=\min \{k-N,[\alpha N]-k\}$, $\gamma_{N, k}=\max \{k-N,[\alpha N]-k\}$, we see that as $N \rightarrow \infty$

$$
\begin{gathered}
K_{1}^{N}+K_{2}^{N}=\sum_{\ell=1}^{\beta_{N, k}} \frac{1}{2 \pi i \ell}\left(\epsilon_{k+\ell}-\epsilon_{k-\ell}\right)+ \\
+o(1)\left(\sum_{\beta_{N, k}+1}^{k-N} \frac{1}{\ell}+\sum_{\beta_{n, k}+1}^{[\alpha N]-k} \frac{1}{\ell}\right)+o(1) \\
\leq \frac{|\nu|}{\pi} \sum_{\ell=1, \ell \neq k}^{\beta_{N, k}} \frac{1}{|k+\ell| k-\ell \mid}+\frac{1}{2 \pi} \sum_{1}^{\beta_{N, k}}\left|r_{k+\ell}\right|+\left|r_{k-\ell}\right| \\
+o(1)\left(1+\log \left(\frac{\overline{k-N}}{N, k}\right)+\log \left(\frac{[\alpha N]-k}{\beta_{N, k}+1}\right)\right)
\end{gathered}
$$

uniformly for $k>0, N<k<[\alpha N]-1$, as $N \rightarrow \infty$. In view of (4.63) and (4.64), for $\delta_{1}>0$ arbitrarily small and $N$ large enough, we have that

$$
\left|S_{N, K}\right| \leq C\left(\frac{k-N}{\beta_{N, k}+1}\right)^{\delta_{1}}\left(\frac{[\alpha N]-k}{\beta_{N, k}+1}\right)^{\delta_{1}} \leq C|k|^{2 \delta_{1}}
$$

whereupon $\left|L_{N}\right|=o(1)$ as $N \rightarrow \infty$. Finally, minor modifications of the previous argument enable us to remove the assumptions made on the relations between $k$ and $N$, and (4.62) follows.

We next set out to derive (4.56). To this end we notice that, keeping to our previous notation 


$$
\tilde{M}_{0}^{N}=\sum_{\infty}^{\infty} 2 \pi i k \mu_{N, k} \Gamma_{k}+\sum_{N \leq|k| \leq \alpha N} 2 \pi \mathrm{ikR}_{N, k} \Gamma_{k} \equiv B_{1}^{N}+B_{2}^{N}
$$

Arguing as in the previous case, we readily see that

$$
\lim _{N \rightarrow \infty} B_{1}^{N}=M_{0}
$$

Therefore the proof will be finished as soon as can show that

$$
\lim _{N \rightarrow \infty} B_{2}^{N}=-\chi_{0} \nu \log \alpha
$$

To derive (4.67b), we write $B_{2}^{N}$ in the form

$$
\begin{gathered}
B_{2}^{N}=\left(\overline{\left.\sum_{N}^{[\alpha, N]} 2 \pi i k R_{N, k} \Gamma_{k}\right)}+\left(\overline{\left(\sum_{N}^{[\alpha, N]} 2 \pi i k R_{N, k} \Gamma_{k}\right.}\right)\right. \\
\equiv Q_{N}+\bar{Q}_{N}
\end{gathered}
$$

We know have that, as $N \rightarrow \infty$,

$$
\begin{gathered}
Q_{N}=-\chi_{0} \sum_{N+1}^{[\alpha N]-1} \epsilon_{k} \delta_{N, k}+o(1) \\
=-\chi_{0} \nu \sum_{N+1}^{[\alpha N]-1} \frac{\delta_{N, k}}{k}-\chi_{0} \sum_{N+1}^{[\alpha N]-1} r_{k} S_{N, k}+o(1) \\
\equiv-\chi_{0} \nu S_{1}+o(1) .
\end{gathered}
$$

On the other hand, for $1<\theta_{1}<\theta_{2}<\alpha$ with $\theta_{1}, \theta_{2}$ close enough to 1 and $\alpha$ respectively, we see that

$$
\begin{gathered}
\left|S_{1}\right| \leq \sum_{N+1}^{\left[\theta_{1} N\right]-1} \frac{\left|S_{N, k}\right|}{k}+\sum_{N+1}^{\left[\theta_{2} N\right]} \frac{\left|S_{N, k}\right|}{k}+ \\
\quad+\sum_{N+1}^{[\alpha N]-1} \frac{\left|S_{N, k}\right|}{k} \equiv \\
\equiv S_{1,1}+S_{1,2}+S_{1,3}
\end{gathered}
$$


Recalling (4.65), it follows at once that, for some $C>0$

$$
\begin{gathered}
S_{1,1} \leq \frac{C}{N} \sum_{N+1}^{\left[\theta_{1} N\right]-1}\left(\frac{k}{N}\right)^{-1}\left(\frac{k}{N}-1\right)^{-\delta_{1}} \leq \\
\leq C \int_{1}^{\theta_{1}} \frac{d r}{r(r-1)^{\delta_{1}}}
\end{gathered}
$$

In a similar way, we obtain that

$$
S_{1,2} \leq C \int_{\theta_{2}}^{\alpha} \frac{d r}{r(r-1)^{\delta_{1}}}
$$

Finally, one readily checks that $S_{N, k}=1+o(1)$ as $N \rightarrow \infty$, uniformly for $k \in\left(\theta_{1} N, \theta_{2} N\right)$. Hence

$$
S_{1,3}=\sum_{\left[\theta_{1}, N\right]}^{\left[\theta_{2}, N\right]} \frac{1}{k}(1+o(1))=\log \left(\frac{\theta_{2}}{\theta_{1}}\right)+o(1) \text { as } N \rightarrow \infty .
$$

Therefore, letting $\theta_{1} \rightarrow 1$ and $\theta_{2} \rightarrow \infty$ in (4.70), we deduce that $S_{1} \rightarrow \log \alpha$ as $N \rightarrow \infty$. From this and (4.68), (4.67), follows.

\section{End of the proof of Theorem 4.12.}

By Lemma 4.13 we can select $f$ ant $T_{w}$ so that an arbitrary (but finite) number of zeroes of the corresponding function $L(z)$ is prescribed at will. Let $S_{R}=\left\{z_{1}, . ., \tilde{z}_{N}\right\}$ be the real roots of $L(z)$ and assume that $\tilde{z}_{N}>\frac{1}{\epsilon}$ and $\tilde{z}_{N}>\left|\tilde{z}_{j}\right|$ for any $\tilde{z}_{j} \in S_{R}$ with $h \neq \mathrm{N}$. Let $\xi_{N}^{\ell}$ be one of the roots of $(3.14 \mathrm{~b})$ with $z_{j}$ replaced by $\tilde{z}_{N}$ there. Clearly, we may assume that $\xi_{N}^{\ell}>0$ and $g^{\prime}\left(\xi_{N}^{\ell}\right)<0$. Furthermore, it may also be supposed that $L\left( \pm \frac{1}{\epsilon}\right)>0$ if roots $\tilde{z}_{j}$ are located in a suitable way.

It is easy to see that we can have that $\chi_{0} \neq 0$ and $M_{0} \neq 0$, after possibly a slight perturbation in the location of the zeroes above. Indeed, suppose that we change the coefficient $\Gamma_{k}, \Gamma_{-k}$ in $L(z)$, where $\Gamma_{k}=a_{k} \bar{b}_{k}$, into $\Gamma_{k}+\Delta \Gamma_{k}, \Gamma_{-k}+\Delta \Gamma_{-k}$. Then $\chi_{0}$ would go into $\tilde{\chi}_{0}=\chi_{0}+\Delta \chi_{0}=\chi_{0}+2 \operatorname{Re}\left(\Gamma_{k}\right)$, and $M_{0}$ would be transformed into $\tilde{M}_{0}=$ $M_{0}-4 \pi k \operatorname{Im}\left(\Gamma_{k}\right)$, whence the result. Indeed this change can be done so that it also yields (4.17). On the other hand, by (4.16) we see that, as $R e \rightarrow \infty$ 


$$
Q z^{2} \chi_{0} \approx\left(\frac{z^{2} R e^{2}}{g\left(\xi_{N}^{\ell}\right)^{2}}\right) \cdot \frac{g^{\prime}\left(\xi_{N}^{\ell}\right) \chi_{0}}{\xi_{N}^{\ell}}
$$

Therefore, by selecting coefficients so that $\chi_{0}>0$, the first term in the right of (4.20) yields purely imaginary values, whereas the second one there behaves as

$$
\frac{1}{2}\left(\frac{\epsilon z^{2} \xi_{N}^{\ell} g^{\prime}\left(\xi_{N}^{\ell}\right)}{\left(g\left(\xi_{N}^{\ell}\right)\right)^{2}}+\frac{M_{0}}{\chi_{0}}\right)
$$

which by (4.55), (4.56) can approach any arbitrary real value if coefficients $\Gamma_{k}$ with $N \leq|k| \leq \alpha N(\alpha>1$, and $N$ large enough) are suitably selected.

\section{STABILITY OF LINEARLY STABLE SOLUTIONS}

In this Section we analyze the local stability of the stationary solutions for the complete nonlinear problem (4.2), (4.3). Assume that $\left(\Phi_{s}, T_{s}\right)$ is a stationary solution as in Section 4. We then define

$$
\begin{aligned}
w & =\Phi(s)-\Phi_{s} \\
\psi(x, s) & =T(x, s)-T_{s}(x)
\end{aligned}
$$

Assume $\Phi_{s} \not \equiv 0$ that is generically true. By (4.2), (4.3) we obtain that as long as $|w|$ is small enough,

$$
\begin{aligned}
\frac{\partial \psi}{\partial s} & +\frac{\varepsilon \operatorname{sgn}\left(\Phi_{s}\right)}{g\left(\operatorname{Re}\left|v_{s}\right|\right)} \frac{\partial \psi}{\partial x}=-\psi+ \\
& +\varepsilon \operatorname{sgn}\left(\Phi_{s}\right) \frac{\partial T_{s}}{\partial x}(x)\left\{\frac{1}{g\left(\operatorname{Re}\left|v_{s}\right|\right)}-\frac{1}{g(\operatorname{Re}|v(s)|)}\right\}+ \\
& +\varepsilon \operatorname{sgn}\left(\Phi_{s}\right)\left\{\frac{1}{g\left(\operatorname{Re}\left|v_{s}\right|\right)}-\frac{1}{g(\operatorname{Re}|v(s)|)}\right\} \frac{\partial \psi}{\partial x}(x, s) \\
\frac{d w}{d s}+ & +\left(\frac{1}{g(\operatorname{Re}|v(s)|)}-\frac{1}{g\left(\operatorname{Re}\left|v_{s}\right|\right)}\right) \oint f(x) T_{s}(x) d x+ \\
& +\left(\frac{1}{g(\operatorname{Re}|v(s)|)}-\frac{1}{g\left(\operatorname{Re}\left|v_{s}\right|\right)}\right) \oint f(x) \psi(x, s) d x
\end{aligned}
$$

where by $(4.1 \mathrm{a}) v_{s}=\operatorname{sgn}\left(\Phi_{s}\right)\left(2\left|\Phi_{s}\right|\right)^{1 / 2}, v(s)=\operatorname{sgn}(\Phi(s))(2|\Phi(s)|)^{1 / 2}$. By $(5.3)$ we have 
that

$$
\begin{aligned}
& \psi(x, s)=e^{-s} \psi_{0}\left(x-\int_{0}^{s} \frac{\varepsilon \operatorname{sgn}\left(\Phi_{s}\right) d \lambda}{g(\operatorname{Re}|v(\lambda)|)}\right)+ \\
&+ \int_{0}^{s} e^{-(s-\lambda)} \varepsilon \operatorname{sgn}\left(\Phi_{s}\right)\left\{\frac{1}{g\left(\operatorname{Re}\left|v_{s}\right|\right)}-\frac{1}{g(\operatorname{Re}|v(\lambda)|)}\right\} \\
& \frac{\partial T_{s}}{\partial x}\left(x-\int_{\lambda}^{s} \frac{\varepsilon \operatorname{sgn}\left(\Phi_{s}\right)}{g(\operatorname{Re}|v(\Phi)|)} d \xi\right) d \lambda
\end{aligned}
$$

Plugging (5.5) into (5.4) we obtain the differential equation

$$
\begin{aligned}
\frac{d w}{d s} & +2 w=\frac{e^{-s}}{g(\operatorname{Re}|v(s)|)} \oint f(x) \psi_{0}\left(x-\int_{0}^{s} \frac{\varepsilon \operatorname{sgn}\left(\Phi_{s}\right)}{g(\operatorname{Re}|v(\lambda)|)} d \lambda\right) \\
& +\int_{0}^{s} \frac{\varepsilon \operatorname{sgn}\left(\Phi_{s}\right)}{g(\operatorname{Re}|v(\lambda)|)} e^{-(s-\lambda)} \oint f(x) \frac{\partial T_{s}}{\partial_{x}}\left(x-\int_{\lambda}^{s} \frac{\varepsilon \operatorname{sgn}\left(\Phi_{s}\right)}{g(\operatorname{Re}|v(s)|)} \lambda \xi\right) d x d x \\
& +\left(\frac{1}{g(\operatorname{Re}|v(s)|)}-\frac{1}{g\left(\operatorname{Re}\left|v_{0}\right|\right)}\right) \oint f(x) T_{0}(x) d x
\end{aligned}
$$

We make the change at variables $w(s)=e^{-s} g(s)$. Then $g(s)$ satisfies the equation

$$
\begin{aligned}
\frac{d q}{d s} & +q=\frac{1}{g(\operatorname{Re}|v(s)|)} \oint f(x) \psi_{0}\left(x-\int_{0}^{s} \frac{\varepsilon \operatorname{sgn}\left(\Phi_{s}\right)}{g|\operatorname{Re}| x(\lambda) \mid)} d \lambda\right) \\
& +\int_{0}^{s} e^{\lambda} \frac{\varepsilon \operatorname{sgn}\left(\Phi_{e}\right)}{g(\operatorname{Re}|v(\lambda)|)}\left(\oint f(x) \frac{\partial T_{s}}{\partial x}\left(x-\int_{\lambda}^{s} \frac{\varepsilon \operatorname{sgn}\left(\Phi_{\varepsilon}\right)}{g|\operatorname{Re}| v(s) \mid)} d \xi\right) d x\right) d \lambda \\
& +e^{s}\left(\frac{1}{g|\operatorname{Re}| v(e))}-\frac{1}{g(\operatorname{Re}|v(s)|)}\right) \oint f(x) T_{s}(x) d x
\end{aligned}
$$

We now define a new time scale as:

$$
\eta \equiv \int_{0}^{Z(\eta)} \frac{g\left(\operatorname{Re}\left|v_{s}\right|\right)}{g|\operatorname{Re}| v(\eta) \mid)} d \lambda, \quad Z(\eta)=s
$$

It is readily seen that $\eta \rightarrow \infty$ as $s \rightarrow \infty$.

On the other hand, (5.6) may be written as 


$$
\begin{aligned}
\frac{d q}{d \eta} & +\frac{g(\operatorname{Re}|v(s)|)}{g\left(\operatorname{Re}\left|v_{s}\right|\right)} q= \\
& =\frac{1}{g\left(\operatorname{Re}\left|v_{s}\right|\right)} \oint f(x) \psi_{0}\left(x-\frac{\varepsilon \operatorname{sgn}\left(\Phi_{s}\right)}{g\left(\operatorname{Re}\left|v_{s}\right|\right)}\right) d x+ \\
& +\frac{\varepsilon \operatorname{sgn}\left(\Phi_{s}\right)}{g\left(\operatorname{Re}\left|v_{s}\right|\right)}\left(\frac{g(\operatorname{Re}|v(s)|)}{g\left(\operatorname{Re}\left|v_{s}\right|\right)}\right) \int_{0}^{\eta} e^{Z(\gamma)} Q \omega|Z(\gamma)| \tilde{K}(\eta-\gamma) d \gamma \\
& +\frac{\varepsilon \operatorname{sgn}\left(\Phi_{s}\right)}{g\left(\operatorname{Re}\left|v_{s}\right|\right)}\left(\frac{g(\operatorname{Re}|v(s)|)}{g\left(\operatorname{Re}\left|v_{s}\right|\right)}\right) \int_{0}^{\eta} e^{Z(\gamma)}\left\{\frac{1}{g\left(\operatorname{Re}\left|v_{s}\right|\right)}-\frac{1}{g(\operatorname{Re}|v(\lambda)|)}-\right. \\
& \quad Q w(Z(\gamma))\} \tilde{K}(\eta-\gamma) d \gamma+ \\
& +e^{Z(\eta)}\left(\frac{1}{g\left(\operatorname{Re}\left|v_{s}\right|\right)}-\frac{1}{g(\operatorname{Re}|s(s)|)}\right) \oint f(x) T_{s}(x) d x
\end{aligned}
$$

where

$$
\tilde{K}(\xi)=\oint f(x) \frac{\partial T_{s}}{\partial x}\left(x-\frac{\xi}{g\left(\operatorname{Re}\left|v_{s}\right|\right)}\right) d x
$$

and $Q$ has been defined in (4.16).

Set

$$
\Gamma \equiv-Q \oint f(x) T_{s}(x) d x
$$

We can transform (5.8) in

$$
\begin{aligned}
& \frac{d q}{d \eta}+(1+\Gamma) q=\frac{1}{g\left(\operatorname{Re}\left|v_{s}\right|\right)} \oint f(x) \psi_{0}\left(x-\frac{\varepsilon \operatorname{sgn}\left(\Phi_{s}\right)}{g\left(\operatorname{Re}\left|v_{s}\right|\right)} \eta\right) d x+ \\
& +\frac{Q \varepsilon \operatorname{sgn}\left(\Phi_{s}\right)}{g\left(\operatorname{Re}\left|v_{s}\right|\right)} \int_{0}^{\eta} \tilde{K}(\eta-\gamma) q(Z(\gamma)) d \gamma+ \\
& +\frac{\varepsilon \operatorname{sgn}\left(\Phi_{s}\right)}{g\left(\operatorname{Re}\left|v_{s}\right|\right)}\left(\frac{g(\operatorname{Re}|v(s)|)}{g\left(\operatorname{Re}\left|v_{s}\right|\right)}\right) \int_{0}^{\eta} e^{Z(\gamma)}\left\{\frac{1}{g\left(\operatorname{Re}\left|v_{s}\right|\right)}-\frac{1}{g(\operatorname{Re}|v(Z(\gamma))|)}-\right. \\
& +\frac{Q \varepsilon \operatorname{sgn}\left(\Phi_{s}\right)}{g\left(\operatorname{Re}\left|v_{s}\right|\right)}\left(\frac{g(\operatorname{Re}|v(s)|)-g\left(\operatorname{Re}\left|v_{s}\right|\right)}{g\left(\operatorname{Re}\left|v_{s}\right|\right)}\right) \int_{0}^{\eta} q(Z(\gamma)) \tilde{K}(\eta-\gamma) d \gamma- \\
& \quad-\left(\frac{g(\operatorname{Re}|v(s)|)-g\left(\operatorname{Re}\left|v_{s}\right|\right)}{g\left(\operatorname{Re}\left|v_{s}\right|\right)}\right) q(s)+ \\
& +e^{Z(q)}\left\{\frac{1}{g\left(\operatorname{Re}\left|v_{0}\right|\right)}-\frac{1}{g(\operatorname{Re}|v(s)|)}-Q w(s)\right\} \oint f(x) T_{s}(x) d x
\end{aligned}
$$


Define

$$
\begin{aligned}
& \Omega(\xi) \equiv e^{-\eta} q(Z(\eta)) \\
& K(\xi)=e^{-\xi} \tilde{K}(\xi)
\end{aligned}
$$

Then, we have

$$
\begin{aligned}
\frac{d \Omega}{d \eta} & +(2+\Gamma) \Omega=\frac{e^{-\eta}}{g\left(\operatorname{Re}\left(v_{s}\right)\right)} \oint f(x) \psi_{0}\left(x-\frac{\varepsilon \operatorname{sgn}\left(\Phi_{s}\right)}{g\left(\operatorname{Re}\left(v_{s}\right)\right)} \eta\right) d x \\
& +\frac{Q \varepsilon \operatorname{sgn}\left(\Phi_{s}\right)}{g\left(\operatorname{Re}\left|v_{s}\right|\right)} \int_{0}^{\eta} K(\eta-\gamma) \Omega(\gamma) d \gamma+ \\
& +\frac{\varepsilon \operatorname{sgn}\left(\Phi_{s}\right)}{g\left(\operatorname{Re}\left|v_{s}\right|\right)}\left(\frac{g(\operatorname{Re}|v(s)|)}{g\left(\operatorname{Re}\left|v_{s}\right|\right)}\right) \int_{0}^{\eta} e^{-\eta} e^{Z(\gamma)}\left\{\frac{1}{g\left(\operatorname{Re}\left|v_{s}\right|\right)}-\frac{1}{g(\operatorname{Re}|v(\gamma)|)}-\right. \\
& +\frac{Q \varepsilon \operatorname{sgn}\left(\Phi_{s}\right)}{g\left(\operatorname{Re}\left(v_{s}\right)\right)}\left(\frac{g(\operatorname{Re}|v(s)|)-g\left(\operatorname{Re}\left|v_{s}\right|\right)}{g\left(\operatorname{Re}\left|v_{s}\right|\right)}\right) \int_{0}^{\eta} K(\eta-\gamma) \Omega(\gamma) d \gamma \\
& -\left(\frac{g(\operatorname{Re}|v(s)|)-g\left(\operatorname{Re}\left|v_{s}\right|\right)}{g\left(\operatorname{Re}\left|v_{s}\right|\right)}\right) \Omega(\eta)+ \\
& +e^{-\eta} e^{Z(\eta)}\left\{\frac{1}{g\left(\operatorname{Re}\left|v_{s}\right|\right)}-\frac{1}{g(\operatorname{Re}|v(s)|)}-Q \omega(s)\right\} \oint f(x) T_{s}(x) d x
\end{aligned}
$$

Our goal is to analyze equation (5.10) for $|\Omega(0)|\left\|\psi_{0}()\right\|_{H_{p}^{1}(C)}$ small. To this end, we first study the linear integrodifferential equation

$$
\frac{d \Omega}{d \eta}+(2+\Gamma) \Omega=\frac{Q \varepsilon \operatorname{sgn}\left(\Phi_{s}\right)}{g\left(\operatorname{Re}\left|v_{s}\right|\right)} \int_{0}^{\eta} K(\eta-\gamma) \Omega(\gamma) d \gamma
$$

Suppose that the roots of the linearized problem, that have been analyzed in Section 4, are placed at $J=\left\{\mu_{k} \in \mathbb{C}: k=0, \pm 1, \pm 2 \ldots\right\}$. Then

Lemma 5.1. Assume that $-1<\tau<0, \operatorname{Re}\left\{\mu_{k}\right\}<\tau$ for $\mu_{k} \in J$. The problem (5.13) with the initial condition $\Omega(0)=\Omega_{0}$ has a unique solution $\Omega(t) \in C[0+\infty) \cap C^{1}(0+\infty)$. This solution satisfies

$$
|\Omega(\eta)| \leq C\left(\Omega_{0}\right) e^{\tau \eta}
$$

where $C>0$ is some constant depending only on $R e,\|f\|_{H_{p}^{\prime}}\left\|T_{w}\right\|_{H_{p}^{1}}, g$.

Proof. Local existence and uniqueness of solutions for the problem $(5.12), \Omega(0)=\Omega_{0}$ follows from a standard fixed point argument. On the other hand by the variation of constants formula

$$
\Omega(\eta)=\Omega_{0} e^{-(2+\Gamma) \eta}+\frac{Q \varepsilon \operatorname{sgn}\left(\Phi_{s}\right)}{g\left(\operatorname{Re}\left|v_{s}\right|\right)} \int_{0}^{\eta} d \xi e^{-(2+\Gamma)(\eta-\xi)}-\int_{0}^{\xi} K(\xi-\gamma) \Omega(\gamma) d \gamma
$$


whence taking into account (3.9), (5.11)

$$
|\Omega(\eta)| \leq\left|\Omega_{0}\right| e^{-(2+\Gamma) \eta}+C \int_{0}^{\eta} d \xi e^{-(2+\Gamma)(\eta-\xi)} \int_{0}^{\xi} e^{-(\xi-\gamma)}|\Omega(\gamma)| d \gamma
$$

where $C>0$ depends on $f, T_{w}, R e$, and $g$. A straightforward calculation gives

$$
|\Omega(\eta)| \leq\left|\Omega_{0}\right| e^{-(2+\Gamma) \eta}+C \int_{0}^{\eta} e^{\tilde{L}(\eta-\gamma)}|\Omega(\gamma)| d \gamma
$$

where $\tilde{L}>0$ and $C>0$ may change from line to line. A continuation argument implies that for $L>0$ large enough

$$
|\Omega(\eta)| \leq C e^{L \eta}
$$

and global existence follows.

By (5.13) we can apply Laplace's transform to (5.13). Set

$$
\hat{f}(z)=\int_{0}^{+\infty} e^{-z t} f(t) d t
$$

to obtain $z \widehat{\Omega}(z)-\Omega_{0}+(2+\Gamma) \widehat{\Omega}(z)=\frac{Q \varepsilon \operatorname{sgn}\left(\Phi_{s}\right)}{g\left(R e\left|v_{s}\right|\right)} \widehat{K}(z) \widehat{\Omega}(z)$. Notice that $\widehat{\Omega}(z)$ is analytic for $\operatorname{Re}(z)>L$. Then

$$
\widehat{\Omega}(z)=\frac{\Omega_{0}}{\left(z+(2+\Gamma)-\frac{Q \varepsilon \operatorname{sgn}\left(\Phi_{s}\right)}{g\left(\operatorname{Re}\left|v_{s}\right|\right)} \widehat{K}(z)\right)}
$$

By the inversion's formula for the Laplace transform

$$
\Omega(\eta)=\frac{1}{2 \pi i} \lim _{R \rightarrow \infty} \int_{-R i+2 L}^{R i+2 L} \frac{\Omega_{0} e^{z \eta}}{\left(z+(2+\Gamma)-\frac{Q \varepsilon \operatorname{sgn}\left(\Phi_{s}\right)}{g\left(\operatorname{Re}\left(v_{s}\right)\right)} \widehat{K}(z)\right)} d z
$$

On the other hand, it is readily seen that the zeroes of $z+(2+\Gamma)-\frac{Q \varepsilon \operatorname{sgn}\left(\Phi_{s}\right)}{g\left(\operatorname{Re}\left(v_{s}\right)\right)} \widehat{K}(z)$ are placed at the same points that the zeroes of $\left(1-e^{z}\right) S(z)(\mathrm{cf} .(4.9))$.

Notice that

$$
\begin{aligned}
\widehat{K}(z) & =\int_{0}^{+\infty} d t e^{-z t} K(t) d t= \\
& =\int_{0}^{+\infty} d t e^{-z t} e^{-t} \oint \frac{\partial T_{s}}{\partial x}\left(x-\frac{\varepsilon \operatorname{sgn}\left(\Phi_{s}\right)}{g\left(\operatorname{Re}\left|v_{s}\right|\right)} t\right) f(x) d x= \\
& =\sum_{k=-\infty} \frac{2 \pi k i \bar{a}_{k} c_{k}}{z+1+2 \pi k i \frac{\varepsilon \operatorname{sgn}\left(\Phi_{s}\right)}{g\left(\operatorname{Re}\left|v_{s}\right|\right)}},
\end{aligned}
$$


where $c_{k}$ is given in (3.7).

It is readily seen that uniformly in

$$
\begin{gathered}
\left|z+1+2 \pi k i \frac{\varepsilon \operatorname{sgn}\left(\Phi_{s}\right)}{g\left(\operatorname{Re}\left|v_{s}\right|\right)}\right| \geq \frac{1}{2}|\pi k| \frac{\varepsilon}{\left|g\left(\operatorname{Re}\left|v_{s}\right|\right)\right|} \\
\widehat{K}(z) \simeq+\frac{\sum_{k=-\infty}^{k=+\infty} 2 \pi k i \bar{a}_{k} c_{k}}{z} \text { as }|z| \rightarrow \infty
\end{gathered}
$$

We now define a sequence of contours $C_{n}$ as in the figure

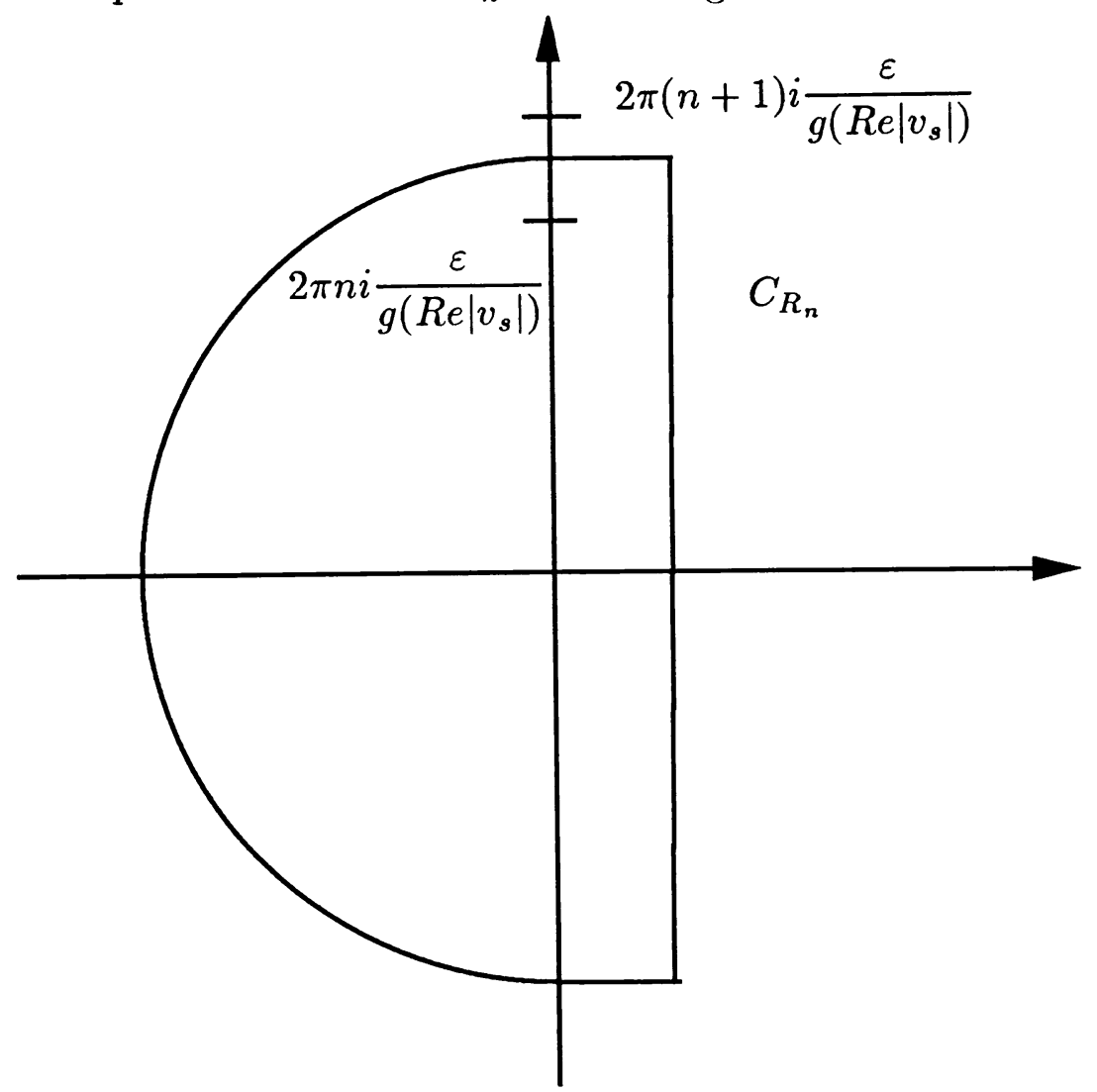

Figure 2:

Then it may be easily seen from (5.16), (5.17) that:

$$
\Omega(\eta)=\frac{\Omega_{0}}{2 \pi i} \lim _{n \rightarrow \infty} \int_{C_{n}} \frac{e^{z \eta}}{\left(z+(2+\Gamma)-\frac{Q \varepsilon \operatorname{sgn}\left(\Phi_{s}\right)}{g\left(\operatorname{Re}\left|v_{s}\right|\right)} \widehat{K}(z)\right)} d z
$$

and by residue theorem we finally arrive to the representation formula

$$
\Omega(\eta)=\lim _{N \rightarrow \infty} \sum_{|\ell| \leq N} \operatorname{Re}\left\{\widehat{\Omega}(z) e^{z \eta}, \mu_{\ell}\right\}
$$


where $\mu_{\ell}$ are the zeroes of $\left(1-e^{z}\right) S(z)$ that have been analyzed in Section 4.

In order to obtain bounds of $\Omega(\eta)$ we need precise estimates on the zeroes of

$$
W(z)=\left(z+(2+\Gamma)-\frac{Q \varepsilon \operatorname{sgn}\left(\Phi_{s}\right)}{g\left(\operatorname{Re}\left|v_{s}\right|\right)} \widehat{K}(z)\right)
$$

Notice that

$$
W(z)=z+(2+\Gamma)+\frac{Q \varepsilon \operatorname{sgn}\left(\Phi_{s}\right)}{g\left(\operatorname{Re}\left(v_{s}\right)\right)} \sum_{k=-\infty}^{k=+\infty} \frac{2 \pi k i \bar{a}_{k} c_{k}}{\left(z+1+2 \pi k i \frac{\varepsilon \operatorname{sgn}\left(\Phi_{i}\right)}{g\left(\operatorname{Re}\left(v_{s}\right)\right)}\right)}
$$

Arguing as in previous sections, we can obtain an asymptotic formula for the roots of $W$. Notice that the equation $W(z)=0$ may be approximated as:

$$
z=\frac{Q \varepsilon \operatorname{sgn}\left(\Phi_{s}\right)}{g\left(\operatorname{Re}\left|v_{s}\right|\right)} \frac{2 \pi j i \bar{a}_{j} c_{j}}{\left(z+1+2 \pi j i \frac{\varepsilon \operatorname{sgn}\left(\Phi_{s}\right)}{g\left(\operatorname{Re}\left|v_{s}\right|\right)}\right)}
$$

Then,

$$
\mu_{j} \simeq-1-2 \pi j i \frac{\varepsilon \operatorname{sgn}\left(\Phi_{s}\right)}{g\left(\operatorname{Re}\left|v_{s}\right|\right)} \text { as }|j| \rightarrow \infty
$$

whence:

$$
(2+\Gamma)-1-2 \pi j i \frac{\varepsilon \operatorname{sgn}\left(\Phi_{s}\right)}{g\left(\operatorname{Re}\left|v_{s}\right|\right)} \simeq \frac{Q \varepsilon \operatorname{sgn}\left(\Phi_{s}\right)}{g\left(\operatorname{Re}\left|v_{s}\right|\right)}-\frac{2 \pi j i \bar{a}_{j} c_{j}}{\left(2+1+\frac{+2 \pi j i \varepsilon \operatorname{sgn}\left(\Phi_{s}\right)}{g\left(\operatorname{Re}\left|v_{s}\right|\right)}\right)}
$$

Then:

$$
\mu_{j} \simeq-\left(1+\frac{2 \pi j i \varepsilon \operatorname{sgn}\left(\Phi_{s}\right)}{g\left(\operatorname{Re}\left|v_{s}\right|\right)}\right)-\frac{Q \varepsilon \operatorname{sgn}\left(\Phi_{\Gamma}\right)}{g\left(\operatorname{Re}\left|v_{s}\right|\right)} \frac{2 \pi j i \bar{a}_{j} c_{j}}{2 \pi j i \frac{\varepsilon \operatorname{sgn}\left(\Phi_{s}\right)}{g\left(\operatorname{Re}\left|v_{s}\right|\right)}}
$$

as $|j| \rightarrow \infty$. Then

$$
\mu_{j} \simeq-\left(1+\frac{2 \pi j i \varepsilon \operatorname{sgn}\left(\Phi_{s}\right)}{g\left(\operatorname{Re}\left|v_{s}\right|\right)}\right)-\bar{a}_{j} c_{j}, \quad \text { as }|j| \rightarrow \infty
$$

Generically we can assume that the roots of $W(z)$ are simple.

Then

$$
\operatorname{Res}\left\{\widehat{\Omega}(z) e^{z \eta}, \mu_{\ell}\right\} \simeq \frac{e^{\mu_{\ell} \tau}}{W^{\prime}\left(\mu_{\ell}\right)}
$$


Notice that

$$
\begin{aligned}
W^{\prime}(z) & =1+\frac{Q \varepsilon \operatorname{sgn}\left(\Phi_{s}\right)}{g\left(R e\left|v_{s}\right|\right)} \sum_{k=-\infty}^{k=+\infty} \frac{2 \pi k i \bar{a}_{k} c_{k}}{\left(z+1+\frac{2 \pi k i \varepsilon \operatorname{sgn}\left(\Phi_{s}\right)}{g\left(R e\left|v_{s}\right|\right)}\right)^{2}} \\
W^{\prime}\left(\mu_{j}\right) & \simeq 1+\frac{Q \varepsilon \operatorname{sgn}\left(\Phi_{s}\right)}{g\left(\operatorname{Re}\left|v_{s}\right|\right)} \cdot \frac{2 \pi j i \bar{a}_{j} c_{j}}{\left(\mu_{j}+1+\frac{2 \pi j_{i} \varepsilon \operatorname{sgn}\left(\Phi_{s}\right)}{g\left(\operatorname{Re}\left|v_{s}\right|\right)}\right)^{2}} \simeq \\
& \simeq 1+\frac{Q \varepsilon \operatorname{sgn}\left(\Phi_{s}\right)}{g\left(\operatorname{Re}\left|v_{s}\right|\right)} \frac{2 \pi j i \bar{a}_{j} c_{j}}{\left(\bar{a}_{j} c_{j}\right)^{2}} \simeq \\
& \simeq \frac{Q \varepsilon \operatorname{sgn}\left(\Phi_{s}\right)}{g\left(\operatorname{Re}\left|v_{s}\right|\right)} \frac{2 \pi j i}{\bar{a}_{j} c_{j}} \quad \text { as }|j| \rightarrow \infty
\end{aligned}
$$

Then, by (5.20)

$$
\operatorname{Res}\left\{\widehat{\Omega}(z) e^{z \eta}: \mu_{l}\right\} \simeq \frac{\Omega_{0} g\left(\operatorname{Re}\left(v_{s}\right)\right)}{Q \varepsilon \operatorname{sgn}\left(\Phi_{s}\right)} \frac{\bar{a}_{l} c_{l}}{2 \pi l i} e^{\mu_{l} \eta}
$$

Then, if we use (5.18) we obtain that $(\Omega(\eta)) \leq C e^{\tau \eta}\left|\Omega_{0}\right|$, where $C$ depends on $f, T_{w}(x)$, and $-1<\tau<0$. Notice that similar bounds may be obtained if $\bar{a}_{l} c_{l}=0$, because $\tau>-1$.

Set $T(\eta)=\Omega(\eta)$ the solution of (5.13) with $\Omega(0)=1$.

We now consider the problem

$$
\begin{aligned}
& \frac{d \Omega}{d \eta}+(2+\Gamma) \Omega=\frac{Q \varepsilon \operatorname{sgn}\left(\Phi_{s}\right)}{g\left(\operatorname{Re}\left(v_{s}\right)\right)} \int_{0}^{\eta} K(\eta-\gamma) \Omega(\gamma) d \gamma+M(\eta) \\
& \Omega(0)=\Omega_{0}
\end{aligned}
$$

where $M(\eta)$ is a continuous function for $\eta \geq 0$. Then, we have

Lemмa 5.2. The problem (5.22) has a unique solution $\Omega(\eta) \in C[0,+\infty)$ that admits the representation formula

$$
\Omega(\eta)=T(\eta) \Omega_{0}+\int_{0}^{\eta} T(\eta-\gamma) M(\eta) d \gamma
$$

Proof. Local existence and uniqueness follows from a standard fixed point argument. By Lemma 5.1 we have that $T(\cdot) \in C[0+\infty) \cap C^{1}(0+\infty)$. Then, a straightforward calculation shows that $\Omega(\eta)$ solves $(5.22 \mathrm{a})$ and $(5.22 \mathrm{~b})$.

Finally we can state the main result of this section. 
ThEOREM 5.3. Assume that there exists $-1<\tau<0$ such that $\operatorname{Re}\left\{\mu_{k}\right\}<\tau$ for $\mu_{k} \in J$. There exists $\delta>0$ such that for $\left|\Omega_{0}\right|+\left\|\psi_{0}\right\|_{H_{p}^{\prime}(C)} \leq \delta$ the solution of (5.12) $(\Omega(\eta), \psi(\eta))$ satisfies

$$
|\Omega(\eta)|+\|\psi(\cdot, \eta)\|_{H_{p}^{1}(C)} \leq C\left(\left|\Omega_{0}\right|+\left\|\psi_{0}\right\|_{H_{p}^{1}(C)}\right) e^{\tau \eta}
$$

where $C>0$ depends on $f, T_{w}, g, R e, \tau$.

Proof. Problem (4.2)-(4.3) is equivalent to (5.5)-(5.12) if $|\Omega(\eta)|$ is small enough. Then global existence and uniqueness of (5.12) follows from Theorem 2.1 and estimate (5.24) if $\delta>0$ is small enough.

Assume that $|\Omega(\eta)| \leq \bar{\delta}$, where $\bar{\delta}$ is small enough. Then, by (5.7) we have that $(1-\beta) \eta \leq Z(\eta) \leq(1+\beta) \eta$, where $\beta>0$ is arbitrarily small. We apply the representation formula (5.23) in (5.17) to obtain

$$
\begin{aligned}
\Omega(\eta) & =T(\eta) \Omega_{0}+ \\
+ & \frac{1}{g\left(\operatorname{Re}\left|v_{s}\right|\right)} \int_{0}^{\eta} d \gamma T(\eta-\gamma) e^{-\gamma} \oint f(x) \psi_{0}\left(x-\frac{\varepsilon \operatorname{sgn}\left(\Phi_{s}\right)}{g\left(\operatorname{Re}\left|v_{s}\right|\right)} \gamma\right) d_{x} \\
+ & \frac{\varepsilon \operatorname{sgn}\left(\Phi_{s}\right)}{g\left(\operatorname{Re}\left|v_{s}\right|\right)} \int_{0}^{\eta} d \gamma T(\eta-\gamma)\left(\frac{g(\operatorname{Re}|v(Z(\gamma))|)}{g\left(\operatorname{Re}\left|v_{s}\right|\right)}\right) \\
& -\int_{0}^{\gamma} e^{-\gamma} e^{Z(\xi)}\left\{\frac{1}{g\left(\operatorname{Re}\left|v_{s}\right|\right)}-\frac{1}{g(\operatorname{Re}|v(\xi)|)}-Q w(Z(\xi))\right\} \widetilde{K}(\gamma-\xi) d \xi \\
+ & \frac{Q \varepsilon \operatorname{sgn}\left(\Phi_{s}\right)}{g\left(\operatorname{Re}\left(v_{s}\right)\right)} \int_{0}^{\eta} d \gamma T(\eta-\gamma)\left(\frac{g(\operatorname{Re}|v(Z(\gamma))|)-g\left(\operatorname{Re}\left(v_{s}\right)\right)}{g\left(\operatorname{Re}\left(v_{s}\right)\right)}\right) \\
& -\int_{0}^{\gamma} K(\gamma-\xi) \Omega(\xi) d \xi- \\
& -\int_{0}^{\eta} d \gamma T(\eta-\gamma)\left(\frac{g(\operatorname{Re}|v(Z(\gamma))|)-g\left(\operatorname{Re}\left(v_{s}\right)\right)}{g\left(\operatorname{Re}\left(v_{s}\right)\right)}\right) \Omega(\gamma)+ \\
+ & \int_{0}^{\eta} d \gamma T(\eta-\gamma) e^{-\gamma} e^{Z(\gamma)}\left\{\frac{1}{g\left(\operatorname{Re}\left(v_{s}\right)\right)}-\frac{1}{g(\operatorname{Re}|v(Z(\gamma))|)}-Q w(Z(0))\right\} \\
\oint & \oint f(x) T_{s}(x) d x
\end{aligned}
$$


Standard estimates that use Lemma 5.1 imply that

$$
\begin{aligned}
|\Omega(\eta)| \leq & C\left(\Omega_{0}\right) e^{\tau \eta}+C\left\|\psi_{0}\right\|_{L^{2}(C)} e^{-\eta}+ \\
& +C \int_{0}^{\eta} d \gamma e^{\tau(\eta-\gamma)} e^{-\gamma} \int_{0}^{\gamma} e^{(1+\beta) \xi}(\Omega(Z(\xi)))^{2} \\
& +C \int_{0}^{\eta} d \gamma e^{\tau(\eta-\gamma)}(\Omega(Z(\gamma))) \int_{0}^{\gamma} e^{-(\gamma-\xi)}(\Omega(\xi)) d \xi+ \\
& +C \int_{0}^{\eta} d \gamma e^{\tau(\eta-\gamma)}(\Omega(Z(\gamma)))(\Omega(\gamma))+ \\
& +C \int_{0}^{\eta} d \gamma e^{\tau(\eta-\gamma)} e^{-\gamma} e^{(1+\beta) \gamma}(\Omega(Z(\gamma))) .
\end{aligned}
$$

Then, if we take into account that as long as (5.24) holds $Z(\eta) \leq(1+\beta) \eta$, we can use a standard continuation argument to obtain that if $\beta$ is small enough

$$
|\Omega(\eta)| \leq C\left(\left|\Omega_{0}\right|+\left\|\psi_{0}\right\|_{L^{2}(C)}\right) e^{\tau \eta}
$$

We now can use (5.5) to obtain

$$
\|\psi(\cdot, \tau)\|_{H_{p}^{1}(C)} \leq C\left(\left|\Omega_{0}\right|+\left\|\psi_{0}\right\|_{H_{p}^{1}(C)}\right) e^{\tau \eta}
$$

This concludes the proof of (5.24).

\section{REFERENCES.}

[C] K. Chen, On the oscillatory instabilities of closed-loop thermosyphons. J. Heat Transfer, Trans. of the ASME, 107 (1985), 826-832.

[H] J.E. Hart, Observations of complex oscillations in a closed thermosyphon. J. Heat Transfer, Trans. of the ASME, 107, (1985), 833-839.

[HV] M.A. Herrero and J.J.L. Velázquez, Stability analysis of a closed thermosyphon. European Jnl. of Applied Maths. 1 (1990), 1-24.

[K] J.B. Keller, Periodic oscillations in a model of thermal convection. J. Fluid Mech. 26,3 (1966), 599-606.

[L] A. Liñán, Analytical description of chaotic oscillations in a toroidal thermosyphon, preprint. 
[PT] L. Prandtl and O.G. Tietjens, Applied Hydro - and Aeromechanics, Ed. Dover (1957).

[SRT] M. Sen, E. Ramos and C. Treviño, The toroidal thermosyphon with known heat flux. Int. J. Heat Mass. Transfer, 28,1 (1985), 219-233.

[W] P. Welander, On the oscillatory instability of a differentially heated fluid loop. J. Fluid Mech. 29 (1967), 17-30. 
1011 E.G. Kalnins, Willard Miller, Jr. and Sanchita Mukherjee, Models of $q$-algebra representations: Matrix Elements of $U_{q}\left(s u_{2}\right)$

Zhangxin Chen and Bernardo Cockburn, Error estimates for a finite element method for the drift-diffusion semiconductor device equations

1014 Richard E. Ewing and Hong Wang, Eulerian-Lagrangian localized adjoint methods for reactive transport in groundwater

1015 Bing-Yu Zhang, Taylor series expansion for solutions of the Korteweg-de Vries equation with respect to their initial values

Kenneth R. Driessel, Some remarks on the geometry of some surfaces of matrices associated with Toeplitz eigenproblems

C.J. Van Duijn and Peter Knabner, Flow and reactive transport in porous media induced by well injection: Similarity solution

Wasin So, Rank one perturbation and its application to the Laplacian spectrum of a graph

G. Baccarani, F. Odeh, A. Gnudi and D. Ventura, A critical review of the fundamental semiconductor equations

1020 T.R. Hoffend Jr., Magnetostatic interactions for certain types of stacked, cylindrically symmetric magnetic particles

IMA Summer Program for Graduate Students, Mathematical Modeling

Wayne Barrett, Charles R. Johnson, and Pablo Tarazaga, The real positive definite completion problem for a simple cycle

Charles A. McCarthy, Fourth order accuracy for a cubic spline collocation method

Martin Hanke, James Nagy, and Robert Plemmons, Preconditioned iterative regularization for I11-posed problems

John R. Gilbert, Esmond G. Ng, and Barry W. Peyton, An efficient algorithm to compute row and column counts for sparse Cholesky factorization

1026 Xinfu Chen, Existence and regularity of solutions of a nonlinear nonuniformly elliptic system arising from a thermistor problem

1027 Xinfu Chen and Weiqing Xie, Discontinuous solutions of steady state, viscous compressible Navier-Stokes equations

1028 E.G. Kalnins, Willard Miller, Jr., and Sanchita Mukherjee, Models of $q$-algebra representations: Matrix elements of the $q$-oscillator algebra

W. Miller, Jr. and Lee A. Rubel, Functional separation of variables for Laplace equations in two dimensions

I. Gohberg and I. Koltracht, Structured condition numbers for linear matrix structures

Xinfu Chen, Hele-Shaw problem and area preserved curve shortening motion

Zhangxin Chen and Jim Douglas, Jr. Modelling of compositional flow in naturally fractured reservoirs

Harald K. Wimmer, On the existence of a least and negative-semidefinite solution of the discrete-time algebraic Riccati equation

Harald K. Wimmer, Monotonicity and parametrization results for continuous-time algebraic Riccati equations and Riccati inequalities

Bart De Moor, Peter Van Overschee, and Geert Schelfhout, $H_{2}$ model reduction for SISO systems

Bart De Moor, Structured total least squares and $L_{2}$ approximation problems

Chjan Lim, Nonexistence of Lyapunov functions and the instability of the Von Karman vortex streets

David C. Dobson and Fadil Santosa, Resolution and stability analysis of an inverse problem in electrical impedance tomography - dependence on the input current patterns

C.N. Dawson, C.J. van Duijn, and M.F. Wheeler, Characteristic-Galerkin methods for contaminant transport with non-equilibrium adsorption kinetics

Bing-Yu Zhang, Analyticity of solutions of the generalized Korteweg-de Vries equation with respect-to their initial values

1042 H.J. Sussmann \& W. Liu, A characterization of continuous dependence of trajectories with respect to the input for control-affine systems

Neerchal K. Nagaraj and Wayne A. Fuller, Least squares estimation of the linear model with autoregressive errors

Karen Rudie \& W. Murray Wonham, Protocol verification using discrete-event systems

Rohan Abeyaratne \& James K. Knowles, Nucleation, kinetics and admissibility criteria for propagating phase boundaries

Gang Bao \& William W. Symes, Computation of pseudo-differential operators

Srdjan Stojanovic, Nonsmooth analysis and shape optimization in flow problem

Miroslav Tuma, Row ordering in sparse $Q R$ decomposition 
Onur Toker \& Hitay Özbay, On the computation of suboptimal $H^{\infty}$ controllers for unstable infinite dimensional systems

1049

1050

1051

1052

1053

1054

1055

1056

1057

1058

1059

1060

1061

1062

1063

1064

1065

1066

1067

1068

1069

1070

1071

1072

1073

1074

1075

1076

1077

1078

1079

1080

1081

1082

1083

1084

1085

1086

1087

1088

1089

1090

1091

1092

1093

Hitay Özbay, $H^{\infty}$ optimal controller design for a class of distributed parameter systems

J.E. Dunn \& Roger Fosdick, The Weierstrass condition for a special class of elastic materials

Bei Hu \& Jianhua Zhang, A free boundary problem arising in the modeling of internal oxidation of binary alloys

Eduard Feireisl \& Enrique Zuazua, Global attractors for semilinear wave equations with locally distributed nonlinear damping and critical exponent

I-Heng McComb \& Chjan C. Lim, Stability of equilibria for a class of time-reversible, $D_{n} x O(2)$-symmetric homogeneous vector fields

Ruben D. Spies, A state-space approach to a one-dimensional mathematical model for the dynamics of phase transitions in pseudoelastic materials

H.S. Dumas, F. Golse, and P. Lochak, Multiphase averaging for generalized flows on manifolds

Bei Hu \& Hong-Ming Yin, Global solutions and quenching to a class of quasilinear parabolic equations

Zhangxin Chen, Projection finite element methods for semiconductor device equations

Peter Guttorp, Statistical analysis of biological monitoring data

Wensheng Liu \& Héctor J. Sussmann, Abnormal sub-Riemannian minimizers

Chjan C. Lim, A combinatorial perturbation method and Arnold's whiskered Tori in vortex dynamics

Yong Liu, Axially symmetric jet flows arising from high speed fiber coating

Li Qiu \& Tongwen Chen, $\mathcal{H}_{2}$ and $\mathcal{H}_{\infty}$ designs of multirate sampled-data systems

Eduardo Casas \& Jiongmin Yong, Maximum principle for state-constrained optimal control problems covered by quasilinear elliptic equations

Suzanne M. Lenhart \& Jiongmin Yong, Optimal control for degenerate parabolic equations with logistic growth

Suzanne Lenhart, Optimal control of a convective-diffusive fluid problem

Enrique Zuazua, Weakly nonlinear large time behavior in scalar convection-diffusion equations

Caroline Fabre, Jean-Pierre Puel \& Enrike Zuazua, Approximate controllability of the semilinear heat equation

M. Escobedo, J.L. Vazquez \& Enrike Zuazua, Entropy solutions for diffusion-convection equations with partial diffusivity

M. Escobedo, J.L. Vazquez \& Enrike Zuazua, A diffusion-convection equation in several space dimensions

F. Fagnani \& J.C. Willems, Symmetries of differential systems

Zhangxin Chen, Bernardo Cockburn, Joseph W. Jerome \& Chi-Wang Shu, Mixed-RKDG finite element methods for the 2-D hydrodynamic model for semiconductor device simulation

M.E. Bradley \& Suzanne Lenhart, Bilinear optimal control of a Kirchhoff plate

Héctor J. Sussmann, A cornucopia of abnormal subriemannian minimizers. Part I: The four-dimensional case

Marek Rakowski, Transfer function approach to disturbance decoupling problem

Yuncheng You, Optimal control of Ginzburg-Landau equation for superconductivity

Yuncheng You, Global dynamics of dissipative modified Korteweg-de Vries equations

Mario Taboada \& Yuncheng You, Nonuniformly attracting inertial manifolds and stabilization of beam equations with structural and Balakrishnan-Taylor damping

Michael Böhm \& Mario Taboada, Global existence and regularity of solutions of the nonlinear string equation

Zhangxin Chen, BDM mixed methods for a nonlinear elliptic problem

J.J.L. Velázquez, On the dynamics of a closed thermosyphon

Frédéric Bonnans \& Eduardo Casas, Some stability concepts and their applications in optimal control problems

Hong-Ming Yin, $\mathcal{L}^{2, \mu}(Q)$-estimates for parabolic equations and applications

David L. Russell \& Bing-Yu Zhang, Smoothing and decay properties of solutions of the Korteweg-de Vries equation on a periodic domain with point dissipation

J.E. Dunn \& K.R. Rajagopal, Fluids of differential type: Critical review and thermodynamic analysis

Mary Elizabeth Bradley \& Mary Ann Horn, Global stabilization of the von Kármán plate with boundary feedback acting via bending moments only

Mary Ann Horn \& Irena Lasiecka, Global stabilization of a dynamic von Kármán plate with nonlinear boundary feedback

Vilmos Komornik, Decay estimates for a petrovski system with a nonlinear distributed feedback

Jesse L. Barlow, Perturbation results for nearly uncoupled Markov chains with applications to iterative methods

Jong-Shenq Guo, Large time behavior of solutions of a fast diffusion equation with source

Tongwen Chen \& Li Qiu, $\mathcal{H}_{\infty}$ design of general multirate sampled-data control systems

Satyanad Kichenassamy \& Walter Littman, Blow-up surfaces for nonlinear wave equations, I

Nahum Shimkin, Asymptotically efficient adaptive strategies in repeated games, Part I: certainty equivalence strategies

Caroline Fabre, Jean-Pierre Puel \& Enrique Zuazua, On the density of the range of the semigroup for semilinear heat equations 